\title{
Numerical and analytical study on the pull-in instability of micro-structure under electrostatic loading
}

\author{
Yin Zhang*, Ya-pu Zhao \\ State Key Laboratory of Nonlinear Mechanics (LNM), Institute of Mechanics, Chinese Academy of Science, \\ Beijing 100080, People's Republic of China
}

Received 27 November 2004; received in revised form 8 December 2005; accepted 8 December 2005

Available online 8 February 2006

\begin{abstract}
The one-mode analysis method on the pull-in instability of micro-structure under electrostatic loading is presented. Taylor series are used to expand the electrostatic loading term in the one-mode analysis method, which makes analytical solution available. The one-mode analysis is the combination of Galerkin method and Cardan solution of cubic equation. The one-mode analysis offers a direct computation method on the pull-in voltage and displacement. In low axial loading range, it shows little difference with the established multi-mode analysis on predicting the pull-in voltages for three different structures (cantilever, clamped-clamped beams and the plate with four edges simply-supported) studied here. For numerical multi-mode analysis, we also show that using the structural symmetry to select the symmetric mode can greatly reduce both the computation effort and the numerical fluctuation.
\end{abstract}

(C) 2006 Elsevier B.V. All rights reserved.

Keywords: Micro-structure; Galerkin method; Pull-in; Beam; Plate; One-mode analysis; Multi-mode analysis

\section{Introduction}

In microelectromechanical systems (MEMS), electrical actuation is widely used as an exciting or control mechanism in electrostatic actuator [1], resonant sensor [2-4] and optical scanner [5]. Electrical actuation mechanism offers many advantages like fast response and simple drive electronics [5]. Microstructure is fabricated by MEMS technology and used in microsensors and microactuators. During the manufacturing, electrostatic loading can be left over to become a pronounced factor affecting the behavior of microbeam in some electrostatic devices [6]. As the microstructure is balanced between electrostatic attractive force and mechanical (elastic) restoring force, both electrostatic and elastic restoring force increase when the electrostatic voltage increases. When the voltage reaches the critical value, the pullin value, pull-in instability happens. Pull-in is the point at which the elastic restoring force can no longer balance the electrostatic force. Further increasing the voltage will cause the structure to have dramatic displacement jump which causes structure

\footnotetext{
* Corresponding author. Tel.: +86 1062648720 ; fax: +86 1062561284 .

E-mail address: zhangyin@1nm.imech.ac.cn (Y. Zhang).
}

collapse and failure. Pull-in instability is a snap-through like behavior and it is saddle-node bifurcation type of instability. Larger maximum displacement can provide the structure better sensitivity and tuning range and a large range of beam deflection can be extremely useful for a wide variety of tuning application [1]. In contrast, pull-in instability restricts the structure maximum displacement. Pull-in instability is also a major structural safety concern for MEMS structural design and test [7-9].

For its importance in MEMS structural safety, pull-in instability is widely studied. Single degree of freedom (DOF) massspring system model is the simplest one used to predict the pullin voltage and maximum displacement [1]. Bochobza-Degani and Nemirovsky $[10,11]$ develop the lumped two DOF model for the pull-in analysis of the structure with bending/torsion coupling. For single DOF model, the stiffness of the continuous system is simplified as one spring constant. For structure like beam or plate, the stiffness is influenced by the axial loading, intrinsic stress gradient [12] and geometric nonlinearity due to large deflection [2]. At the same time, the electrostatic force is dependent on the deflection and this deflection dependence property is responsible for the pull-in instability. How to extract the parameters like the spring stiffness and electrostatic force for one DOF system from the physical continuous system 
determines the accuracy of one DOF modeling. As for the stiffening effect on the structure, the spring is treated as Duffing spring (hardening spring) [13-16]. The nonlinear cubic term in the Duffing spring is due to the mid-plane stretching of the structure and such nonlinear term has pronounced influence on the structure secondary resonance for the dynamic study [16]. Such nonlinear term contribution to the stiffening of the structure is also deflection-dependent for the continuous system. Axial force appears in the governing equation as a linear second order term, which can either stiffen (tensile load) or soften (compressive load) the structure. The axial force influence on the beam pull-in displacement and voltage is investigated by many $[2,3,6,17]$ and is clearly indicated by the ratio of the two dimensionless numbers [17]. Electrostatic force also has the softening effect on the structure stiffness [18]. Pamidighantam et al. [19] derived the close form solution for the beam continuous system with fixed-fixed and fixed-free boundary conditions by introducing the concept of the effective stiffness.

Very similarly, Lobontiu and Garcia [20] use lumped effective inertia and stiffness to derive the close form compliance/stiffness equations for the cantilever structure in atomic force microscopy design. As demonstrated by $\mathrm{Hu}$ et al. [18], there is significant computational difference on the pull-in voltage between lumped model and modal expansion method (Galerkin method) and the experimental results match the results of Galerkin method very well. Meanwhile, using the orthogonality of the mode shapes can greatly reduce the computation effort [18]. Choi and Lovell [6] provide a model on the continuous system, but their model on mechanical restoring force part is linear. Because many MEMS structures may experience large deflection, AbdelRahman et al. [2] provide a refined model which includes the nonlinear mechanical restoring force due to the mid-plane stretching, which stiffens the structure. The models presented here account the influence of both axial force and mid-plane stretching.

For multi-mode analysis [10,11,15,16,21], generally speaking, Newton-Rhapson method is used as the solving method and the gradients are calculated for each iteration step. Relative large computational difference is shown for pull-in voltage between one-mode analysis and two mode analysis [10]. Maybe this is due to the trial function for beam deflection used in their Rayleigh-Ritz method though physically it is also well possible that the second mode shape contributes significantly to the deflection. As for the Galerkin method which uses the structure mode shape as its weight function, Wang et al. [21] show the very good convergence on the computation of the pull-in voltage as the mode number increases. Bochobza-Degani et al. [22] presented an algorithm called displacement iteration pull-in extraction (DIPIE) algorithm. DIPIE algorithm uses a relaxation scheme to avoid the numerical calculation of the electrostatic and mechanical fields gradients. The algorithm iterates until the deflection is converged.

The analytical one-mode analysis presented here does not need any iteration. Rong et al. [23] present an analytical method for pull-in analysis of clamped-clamped multilayer beam. Their method is Rayleigh-Ritz method and assumes one deflection shape function. They derive the two governing equations by enforcing the pull-in conditions that the first and second order derivatives of the system energy functional are zero. In their model, the pull-in voltage and displacement are coupled in the two governing equations. For our analytical one-mode analysis presented here, it directly calculates pull-in voltage and displacement in one governing equation. The accuracy of the one-mode analysis depends on Taylor series expansion on electrostatic force term which has the truncation error. Compared with the Taylor series expansion on electrostatic force done by $\mathrm{Hu}$ et al. [18], our one-mode analysis method keeps more Taylor series expansion terms. In multi-mode analysis, the fact that the slope of voltage-displacement around the pull-in approaches infinity is used as the criterion to determine when pull-in happens [21,24]. Therefore, for the numerical multi-mode analysis, the pull-in displacement can have relatively large numerical fluctuation because any tiny change of electrostatic voltage around pull-in point can cause very large displacement change. That is also why the pull-in displacement is extremely sensitive to the voltage step size around the pull-in point in the numerical multimode analysis. However, there is no such numerical fluctuation in the analytical one-mode analysis. The analytical one-mode analysis offers an efficient, direct and fast method to calculate pull-in voltage and displacement.

In this paper, we first analyze the pull-in instability of single degree of freedom system. The governing equation of single DOF system derived from the balance of mechanical (elastic) restoring and electrostatic forces is a cubic equation. The analytical Cardan solution of a cubic equation is shown here that it can also be used to predicate the pull-in instability. The analytical study on the continuous system is based on the assumption that the first mode shape is the dominant deflection shape and only the first mode shape is used for the discretization. The continuous system is discretized and computed by modal analysis (Galerkin method). Such analytical study is also compared with the numerical multi-modal analysis to show its validity (in certain range) and evaluate its applicability range.

\section{Pull-in instability of single degree of freedom system}

The governing equation for single DOF system shown in Fig. 1 is given as

$k w=\frac{C}{(d-w)^{2}}$

$w$ is the plate displacement, $d$ the gap distance between plate and electrode before actuation, $k$ the spring stiffness and $C$ is a constant given as $C=\varepsilon A V^{2} / 2$ [1] ( $\varepsilon$, permittivity of free space; $A$, plate area; and $V$, voltage.) Left side of Eq. (1) is the elastic restoring force and right side is the electrostatic force. For electrostatic force, the expression above does not account for the fringing fields effect [13,23]. By simple manipulation, Eq. (1) can be rewritten as

$w^{3}-2 \mathrm{~d} w^{2}+\mathrm{d}^{2} w-C_{0}=0$

$C_{0}=C / k$. This is cubic equation and can be analytically solved by Cardan solution [25]. In cubic equation, $Q$ is the parameter 

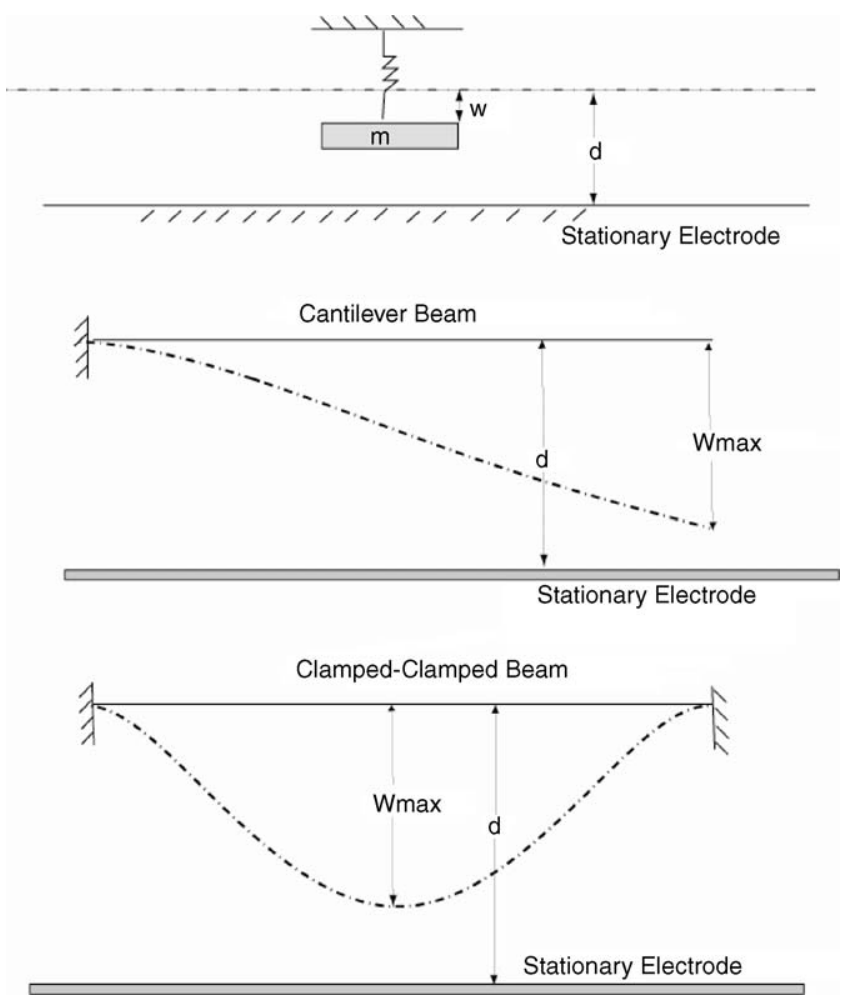

Fig. 1. Schematics of single DOF spring-mass system and cantilever, clamped-clamped beams.

indicating the solution scenarios of the three roots. $Q$ is given as

$Q=\left(\frac{p}{3}\right)^{3}+\left(\frac{q}{2}\right)^{2}$

Here

$p=-\frac{(-2 d)^{2}}{3}+d^{2}=\frac{-d^{2}}{3}$ and

$q=2\left(\frac{-2 d}{3}\right)^{3}-\frac{1}{3}(-2 d) d^{2}-C_{0}=\frac{2 d^{3}}{27}-C_{0}$

When $Q<0$, there are three real roots.

When $Q=0$, there are three real roots, at least two roots are the same.

When $Q>0$, there are one real roots, two complex conjugate roots.

Without loss of generality and for the simplicity, $d$ is set as 1 in Eq. (2). The three roots of Eq. (2) are given in Fig. 2 as $C_{0}$ changes from 0 to $4 / 27$. The three real roots exist until $C_{0}=4 / 27$. At $C_{0}=4 / 27, Q=0$. After $C_{0}=4 / 27$, only $w_{3}$ exists in real domain. $w_{3}$ can be excluded because $w_{3} \geq 1$, which physically means that the plate penetrates the electrode. $w_{2}$ can also be excluded because it is an unstable solution. So only $w_{1}$ is the stable and physically reasonable solution for Eq. (2). From Fig. 2, it is shown that at $C_{0}=4 / 27, w_{1}=w_{2}=1 / 3$. After that, $w_{1}$ and $w_{2}$ cease existing in real domain and only $w_{3}$ is left. Further increasing $C_{0}$, the (real) solution will jump from $w_{1}$ to $w_{3}$ in real domain. This indicates the pull-in instability happening for single DOF case. $1 / 3$ is the maximum plate displacement before

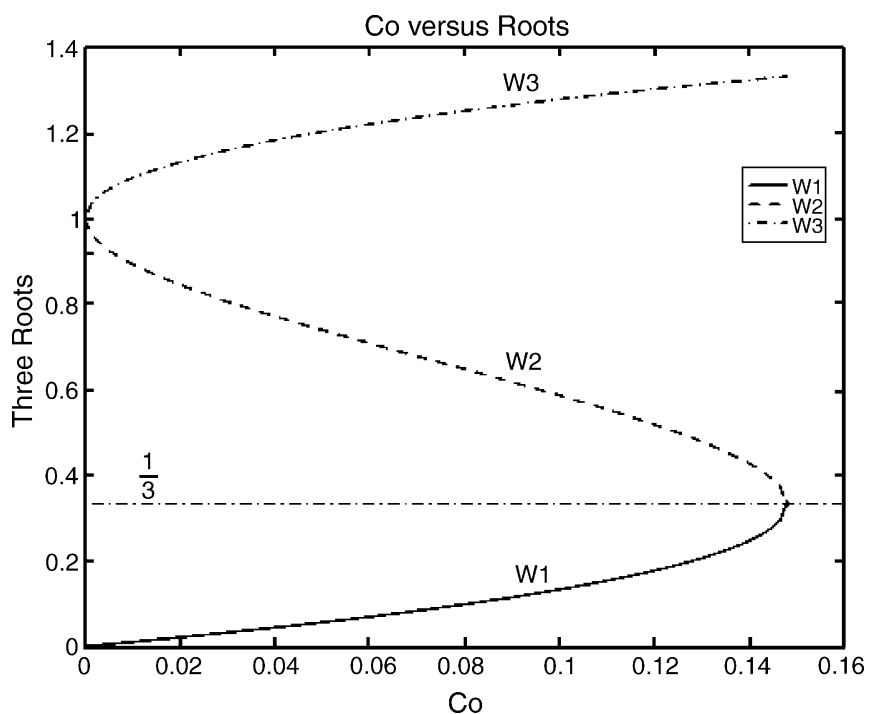

Fig. 2. Three roots change of single DOF system as $C_{0}$ changes.

pull-in instability. This $1 / 3$ value is the widely used criterion among electrical engineers, which is only valid for single DOF system. As it will be shown later, for the continuous system, the axial load will play an important role in this critical value and this $1 / 3$ value can no longer be valid. By analyzing the single DOF case, the conclusion can be made that changing $C_{0}$ results in the change of $Q$ from negative to $0 . Q=0$ indicates pull-in instability. It is also shown that $w_{1}$ and $w_{2}$ are two repeated roots at $Q=0$. This property can also mathematically help to select $w_{1}$ from the three roots at $Q=0$ from Cardan solution.

Another way to look at the problem is to look at the balance between mechanical restoring force $f_{\mathrm{m}}$ and electrical attractive force $f_{\mathrm{e}} . f_{\mathrm{m}}$ is defined as $f_{\mathrm{m}}=k w$ and $f_{\mathrm{e}}$ is defined as $f_{\mathrm{e}}=C /(d-w)^{2}$. Without losing generality and to make things simple, the spring stiffness $k$ is taken as one. In Fig. 3, it is shown

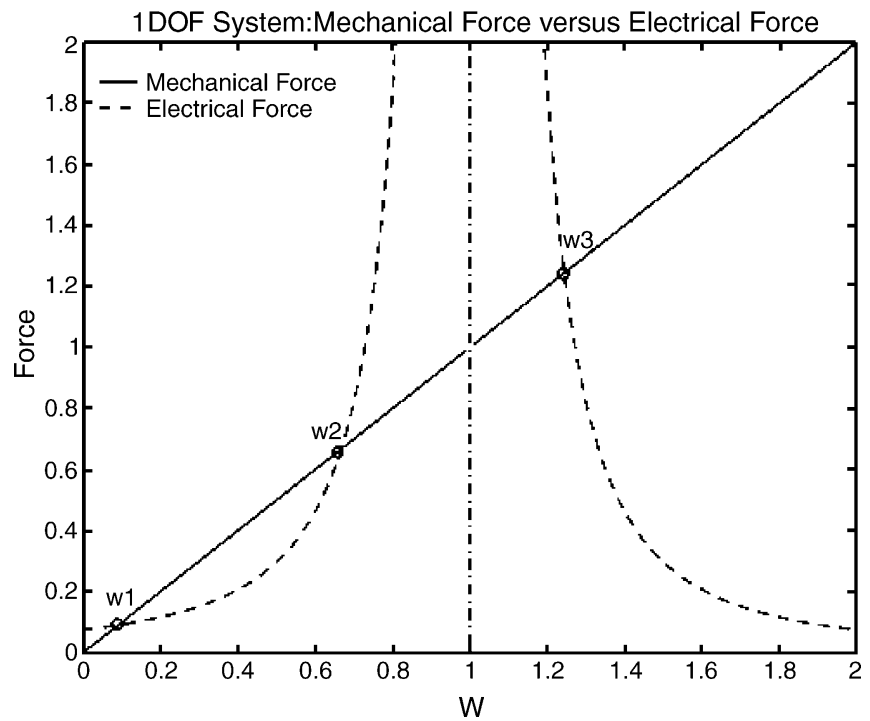

Fig. 3. The balance of mechanical restoring force and electrical force of single DOF system. The spring stiffness $k=1$ and $C=C_{0}=2 / 27$. There are three distinctive intersection points. 


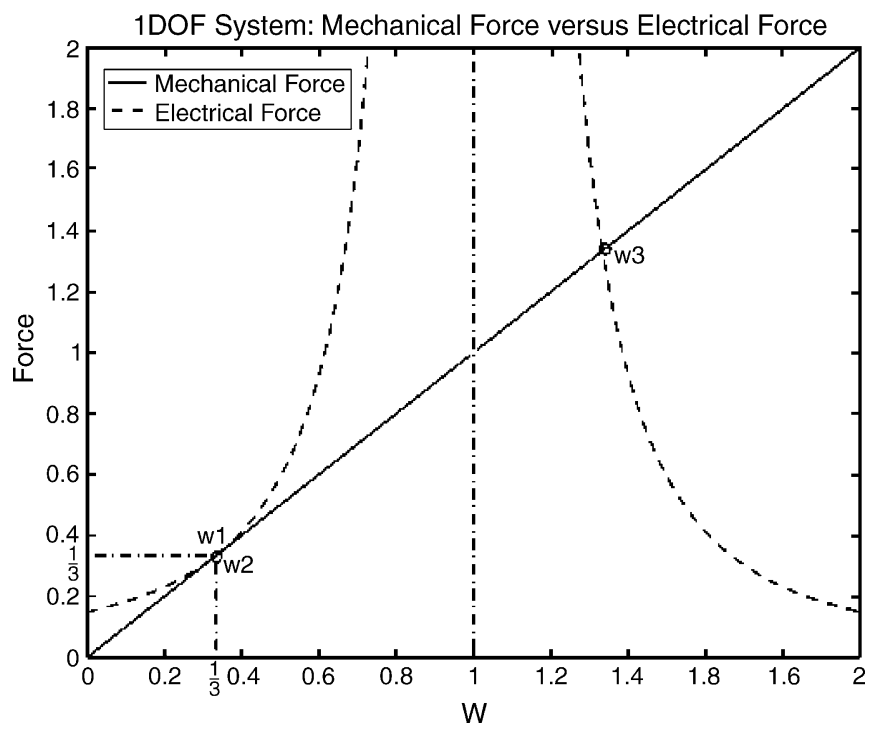

Fig. 4. The balance of mechanical restoring force and electrical force for single DOF system. The spring stiffness $k=1$ and $C=C_{0}=4 / 27$. There are two intersection points. The mechanical force curve is tangent to the electrical force curve and pull-in instability happens.

that when $C=C / k=C_{0}=2 / 27$ (pull-in does not happen), there are three distinctive intersection points, $w_{1}, w_{2}$ and $w_{3}$. These three intersection points are the equilibria of the single DOF system. Clearly $w_{3}\left(w_{3}>1.0\right)$ is physically impossible and $w_{2}$ is an unstable solution. Only $w_{1}$ is the stable and physically reasonable solution. As $C$ increases, $w_{1}$ and $w_{2}$ come closer to each other. As it is shown in Fig. 4, at the point $C=4 / 27$ (pullin happens), the two points collide and the mechanical force curve (the straight line) is tangent to the electrical force curve. If $C$ keeps increasing, there will be no intersection point(s) in $w<1.0$ domain. The only intersection point will be $w_{3}$ and $w_{3}>1.0$, which means there is no physical equilibrium after $C>4 / 27$.

\section{Pull-in instability of continuous system: cantilever and clamped-clamped beams}

\subsection{One-mode analysis of beam}

The nonlinear differential governing equation for electrically actuating beam which accounts for the mid-plane stretching is given as [2]

$E I \frac{\mathrm{d}^{4} w}{\mathrm{~d} x^{4}}=\left[\frac{E A}{2} \int_{0}^{l}\left(\frac{\mathrm{d} w}{\mathrm{~d} x}\right)^{2} \mathrm{~d} x+P\right] \frac{\mathrm{d}^{2} w}{\mathrm{~d} x^{2}}+\frac{\varepsilon b V_{\mathrm{p}}^{2}}{2(d-w)^{2}}$

$E$ is the effective Young's modulus, $I$ the moment of inertia and $I=b h_{3} / 12(b, h$ : beam width and thickness). $A$ the beam cross section area $(A=b h), \varepsilon$ the dielectric constant, $V_{\mathrm{p}}$ the electrostatic voltage, $w$ the beam deflection, $d$ the gap distance and $l$ the beam length. $P$ is the axial load, $P>0$ is tensile and $P<0$ is compressive. For clamped-clamped beam, the boundary condi- tions at the ends are:

$w(0)=0, \quad \frac{\mathrm{d} w(0)}{\mathrm{d} x}=0 ; \quad w(l)=0, \quad \frac{\mathrm{d} w(l)}{\mathrm{d} x}=0$

For cantilever beam, the boundary conditions at the ends are [27]:

$w(0)=0, \quad \frac{\mathrm{d} w(0)}{\mathrm{d} x}=0 ; \quad \frac{d^{2} w(l)}{\mathrm{d} x^{2}}=0$,

$E I \frac{d^{3} w(l)}{\mathrm{d} x^{3}}-P \frac{\mathrm{d} w(l)}{\mathrm{d} x}=0$

Following Abdel-Rahman's nondimensionalization scheme [2], let

$W=\frac{w}{d}, \quad \xi=\frac{x}{l}, \quad N=\frac{P l^{2}}{E I}$,

$\alpha_{1}=6\left(\frac{d}{h}\right)^{2}, \quad \alpha_{2}=\frac{6 \varepsilon l^{4}}{E h^{3} d^{3}}$

Eq. (4) is nondimensionalized as

$\frac{\mathrm{d}^{4} W}{\mathrm{~d} \xi^{4}}=\left[\alpha_{1} \int_{0}^{1}\left(\frac{\mathrm{d} W}{\mathrm{~d} \xi}\right)^{2} \mathrm{~d} \xi+N\right] \frac{\mathrm{d}^{2} W}{\mathrm{~d} \xi^{2}}+\frac{\alpha_{2} V_{\mathrm{p}}^{2}}{(1-W)^{2}}$

To use the analysis of cubic equations, this nonlinear equation needs to have such following manipulation on the electrical loading term

$$
\begin{aligned}
\frac{\alpha_{2} V_{\mathrm{p}}^{2}}{(1-W)^{2}}= & \frac{\alpha_{2} V_{\mathrm{p}}^{2}}{1-\left(2 W-W^{2}\right)} \\
= & \alpha_{2} V_{\mathrm{p}}^{2}\left[1+\left(2 W-W^{2}\right)\right. \\
& \left.+\left(2 W-W^{2}\right)^{2}+\left(2 W-W^{2}\right)^{3}+\cdots\right] \\
\approx & \alpha_{2} V_{\mathrm{p}}^{2}\left(1+2 W+3 W^{2}+4 W^{3}\right)
\end{aligned}
$$

This Taylor series expansion requires $\left|2 W-W^{2}\right|<1$. Because physically $0 \leq W<1$, this requirement can be satisfied. Now Eq. (8) is changed as

$$
\begin{aligned}
\frac{\mathrm{d}^{4} W}{\mathrm{~d} \xi^{4}}= & {\left[\alpha_{1} \int_{0}^{1}\left(\frac{\mathrm{d} W}{\mathrm{~d} \xi}\right)^{2} \mathrm{~d} \xi+N\right] \frac{\mathrm{d}^{2} W}{\mathrm{~d} \xi^{2}} } \\
& +\alpha_{2} V_{\mathrm{p}}^{2}\left(1+2 W+3 W^{2}+4 W^{3}\right)
\end{aligned}
$$

The dimensionless beam deflection $W$ is assumed to have only one mode, $W=a_{1} \phi_{1} . \phi_{1}$ is the first mode shape of cantilever beam or clamped-clamped beam. When the transverse displacement $W$ increases, the Taylor series expansion becomes less accurate because the terms taken in the Taylor series above are only up to cubic term. The mode shapes of the beam with different boundary conditions are given by Chang and Craig [26]. The mode shapes given by Chang and Craig are the mode shapes of the beam without axial loading. For the clamped-clamped beam, the axial load does not change the boundary conditions. For clamped-clamped beam case, the computation results using the mode shapes of zero axial load case make little difference 
First Mode Shapes of Cantilever Beam at Different Axial Load Ns

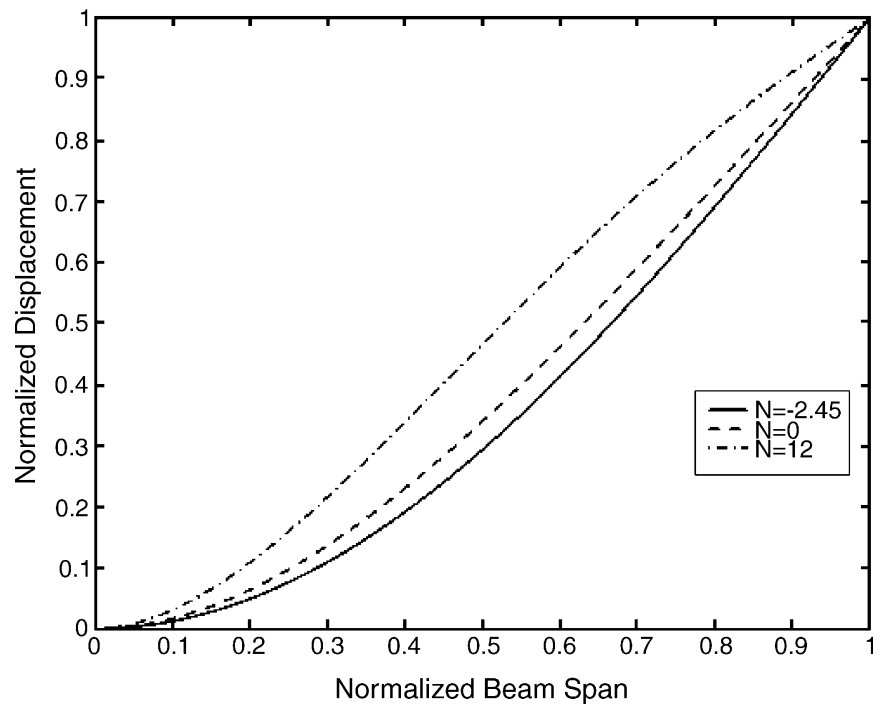

Fig. 5. Different cantilever first mode shapes as the axial load at the end changes.

from those using mode shapes of nonzero axial load when the axial load is moderate. But for cantilever beam, one of boundary condition $\left(E l\left(\mathrm{~d}^{3} w(l) / \mathrm{d} x^{3}\right)-P(\mathrm{~d} w(l) / \mathrm{d} x)=0\right)$ in Eq. (6) is directly related to the axial load $P$. The mode shape for the cantilever beam must be computed every time when the axial load $P(N)$ changes. The computation of the cantilever beam mode shape with axial load is given in the appendix. Fig. 5 shows the changes of the cantilever beam dimensionless first mode shapes under the different axial loads $N$ s.

Substitute $W=a_{1} \phi_{1}$ into Eq. (10), times $\phi_{1}$ and integrate from 0 to 1 . The mode shape $\phi_{1}$ now plays another role of weight function in Galerkin method. Eq. (10) now becomes

$$
\begin{aligned}
& \int_{0}^{1} \phi_{1} \frac{a_{1} \mathrm{~d}^{4} \phi_{1}}{\mathrm{~d} \xi^{4}} \mathrm{~d} \xi \\
& =\int_{0}^{1} \phi_{1}\left[\alpha_{1} \int_{0}^{1}\left(\frac{a_{1} \mathrm{~d} \phi_{1}}{\mathrm{~d} \xi}\right)^{2} \mathrm{~d} \xi+N\right] \frac{\mathrm{d}^{2}\left(a_{1} \phi_{1}\right)}{\mathrm{d} \xi^{2}} \mathrm{~d} \xi \\
& \quad+\int_{0}^{1} \alpha_{2} V_{\mathrm{p}}^{2}\left(\phi_{1}+2 a_{1} \phi_{1}^{2}+3 a_{1}^{2} \phi_{1}^{3}+4 a_{1}^{3} \phi_{1}^{4}\right) \mathrm{d} \xi
\end{aligned}
$$

This is a cubic equation of $a_{1}$ and it can be rewritten as

$a_{1}^{3}+a a_{1}^{2}+b a_{1}+c=0$
Also let

$$
\begin{aligned}
& Q=\left(\frac{p}{3}\right)^{3}+\left(\frac{q}{2}\right)^{2}, \\
& \left(p=-\frac{a^{2}}{3}+b, \quad q=2\left(\frac{a}{3}\right)^{3}-\frac{a b}{3}+c\right)
\end{aligned}
$$

$a, b, c$ contain $\alpha_{2} V_{\mathrm{p}}^{2}$. For pull-in instability, $\alpha_{2} V_{\mathrm{p}}^{2}$ can be determined from the nonlinear equation $Q\left(\alpha_{2} V_{\mathrm{p}}^{2}\right)=0$ as we discuss in one DOF case. Once $\alpha_{2} V_{\mathrm{p}}^{2}$ is solved, it can be substituted into Eq. (12) to solve for $a_{1}$. The maximum displacement of clamped-clamped beam is at the center, so $W_{\max }=a_{1} \phi_{1}(0.5)$. For the cantilever beam, the maximum displacement is at the beam tip, so $W_{\max }=a_{1} \phi_{1}(1.0)$.

There is one problem of solving $\alpha_{2} V_{\mathrm{p}}^{2}$ from the one variable nonlinear equation $Q\left(\alpha_{2} V_{\mathrm{p}}^{2}\right)=0$. Physically there is only one $\alpha_{2} V_{\mathrm{p}}^{2}$ for the pull-in instability to happen. But $Q\left(\alpha_{2} V_{\mathrm{p}}^{2}\right)=0$ of Eq. (15) can give more than one solution. The very intuitive and reasonable choice is to take the smallest positive $\alpha_{2} V_{\mathrm{p}}^{2}$ from the solutions of nonlinear equation $Q\left(\alpha_{2} V_{\mathrm{p}}^{2}\right)=0$. But it happens to be wrong. Remember the physical constraint for clamped-clamped beam requires $W_{\max }=a_{1} \phi_{1}(0.5) \leq 1$. If substitute the smallest $\alpha_{2} V_{\mathrm{p}}^{2}$ calculated from $Q\left(\alpha_{2} V_{\mathrm{p}}^{2}\right)=0$ into Eq. (12) to solve for $a_{1}$, it will make $W_{\max }=a_{1} \phi_{1}(0.5)>1$. When $Q=0$, there are three roots in Eq. (12), two are repeated, $a_{1}$ is taken as the repeated one. It happens to be the second smallest $\alpha_{2} V_{\mathrm{p}}^{2}$ from equation $Q\left(\alpha_{2} V_{\mathrm{p}}^{2}\right)=0$ indicating the pull-in instability of the clamped-clamped beam. For the cantilever beam case, it happens to be the smallest $\alpha_{2} V_{\mathrm{p}}^{2}$ computed from $Q\left(\alpha_{2} V_{\mathrm{p}}^{2}\right)=0$ of Eq. (15) indicating pull-in instability.

\subsection{Multi-mode analysis of beam}

For the multi-mode analysis here, there is no Taylor series expansion operation for the electrical part.

The governing equation is Eq. (8).

$$
\frac{\mathrm{d}^{4} W}{\mathrm{~d} \xi^{4}}=\left[\alpha_{1} \int_{0}^{1}\left(\frac{\mathrm{d} W}{\mathrm{~d} \xi}\right)^{2} \mathrm{~d} \xi+N\right] \frac{\mathrm{d}^{2} W}{\mathrm{~d} \xi^{2}}+\frac{\alpha_{2} V_{\mathrm{p}}^{2}}{(1-W)^{2}}
$$

The displacement is assumed to have the following form

$$
W=\sum_{i=1,2,3 \ldots}^{M} a_{i} \phi_{i}(\xi)
$$

$a, b, c$ are defined as

$a=\frac{3 \alpha_{2} V_{p}^{2} \int_{0}^{1} \phi_{1}^{3} \mathrm{~d} \xi}{G}, \quad b=\frac{2 \alpha_{2} V_{\mathrm{p}}^{2} \int_{0}^{1} \phi_{1}^{2} \mathrm{~d} \xi+N \int_{0}^{1} \phi_{1}\left(\left(\mathrm{~d}^{2}\left(\phi_{1}\right) /\left(\mathrm{d} \xi^{2}\right)\right) \mathrm{d} \xi-\int_{0}^{1} \phi_{1}\left(\left(\mathrm{~d}^{4} \phi_{1}\right) /\left(\mathrm{d} \xi^{4}\right)\right) \mathrm{d} \xi\right.}{G}, \quad c=\frac{\alpha_{2} V_{\mathrm{p}}^{2} \int_{0}^{1} \phi_{1} \mathrm{~d} \xi}{G}$

And $G$ is

$G=4 \alpha_{2} V_{\mathrm{p}}^{2} \int_{0}^{1} \phi_{1}^{4} \mathrm{~d} \xi+\alpha_{1} \int_{0}^{1} \phi_{1} \frac{\mathrm{d}^{2}\left(\phi_{1}\right)}{\mathrm{d} \xi^{2}} \int_{0}^{1}\left(\frac{\mathrm{d} \phi_{1}}{\mathrm{~d} \xi}\right)^{2} \mathrm{~d} \xi \mathrm{d} \xi$ $a_{i}$ is unknown constant (modal amplitude) to be computed from the equation, $\phi_{i}$ is the clamped-clamped beam mode shape or cantilever beam mode shape. The similar Galerkin method is applied here as for one-mode analysis. The final equation set (Eq. (17) shown below) is $M$ coupled polynomial equations for $M a_{i} s(i=1$ to $M) . M$ is the mode number. Newton-Rhapson 
method is used to solve such equation set.

$$
\left\{\begin{array}{l}
\int_{0}^{1} \phi_{1}\left\{\sum_{i=1}^{M} a_{i} \phi_{i}^{\prime \prime \prime \prime}-\left[\alpha_{1} \int_{0}^{1}\left(\sum_{i=1}^{M} a_{i} \phi_{i}^{\prime}\right)^{2} \mathrm{~d} \xi+N\right] \sum_{i=1}^{M} a_{i} \phi_{i}^{\prime \prime}-\frac{\alpha_{2} V_{\mathrm{p}}^{2}}{\left(1-\sum_{i=1}^{M} a_{i} \phi_{i}\right)^{2}}\right\} \mathrm{d} \xi=0 \\
\int_{0}^{1} \phi_{2}\left\{\sum_{i=1}^{M} a_{i} \phi_{i}^{\prime \prime \prime \prime}-\left[\alpha_{1} \int_{0}^{1}\left(\sum_{i=1}^{M} a_{i} \phi_{i}^{\prime}\right)^{2} \mathrm{~d} \xi+N\right] \sum_{i=1}^{M} a_{i} \phi_{i}^{\prime \prime}-\frac{\alpha_{2} V_{\mathrm{p}}^{2}}{\left(1-\sum_{i=1}^{M} a_{i} \phi_{i}\right)^{2}}\right\} \mathrm{d} \xi=0 \\
\vdots \int_{0}^{1} \phi_{M-1}\left\{\sum_{i=1}^{M} a_{i} \phi_{i}^{\prime \prime \prime \prime}-\left[\alpha_{1} \int_{0}^{1}\left(\sum_{i=1}^{M} a_{i} \phi_{i}^{\prime}\right)^{2} \mathrm{~d} \xi+N\right] \sum_{i=1}^{M} a_{i} \phi_{i}^{\prime \prime}-\frac{\alpha_{2} V_{\mathrm{p}}^{2}}{\left(1-\sum_{i=1}^{M} a_{1} \phi_{i}\right)^{2}}\right\} \mathrm{d} \xi=0 \\
\int_{0}^{1} \phi_{M}\left\{\sum_{i=1}^{M} a_{i} \phi_{i}^{\prime \prime \prime \prime}-\left[\alpha_{1} \int_{0}^{1}\left(\sum_{i=1}^{M} a_{i} \phi_{i}^{\prime}\right)^{2} \mathrm{~d} \xi+N\right] \sum_{i=1}^{M} a_{i} \phi_{i}^{\prime \prime}-\frac{\alpha_{2} V_{\mathrm{p}}^{2}}{\left(1-\sum_{i=1}^{M} a_{i} \phi_{i}\right)^{2}}\right\} \mathrm{d} \xi=0
\end{array}\right.
$$

\section{Comparative study of the pull-in instability of the beams under one-mode and multi-mode analysis}

Fig. 6 shows the maximum $\alpha_{2} V_{\mathrm{p}}^{2}$ can be reached before pullin instability happens for the different nondimensional axial force $N$ for the clamped-clamped beam. Fig. 7 shows the same thing for the cantilever beam. $\alpha_{1}$ for both beam structures here is taken as 3.7. In both figures, the one-mode analysis results are compared with the multi-mode analysis results. In the multimode analysis for both the clamped-clamped and cantilever beams, $M$ here is taken as 5 , which shows very good convergence. The critical pull-in $\alpha_{2} V_{\mathrm{p}}^{2}$ increases almost linearly as axial load $N$ increases. The one-mode analysis shows very accurate prediction on the pull-in $\alpha_{2} V_{\mathrm{p}}^{2}$. Fig. 8 shows the critical maximum displacement $(W(0.5))$ at the pull-in $\alpha_{2} V_{\mathrm{p}}^{2}$ for the clamped-clamped beam. Fig. 9 shows the critical maximum displacement $(W(1.0))$ at the pull-in $\alpha_{2} V_{\mathrm{p}}^{2}$ for the cantilever beam.

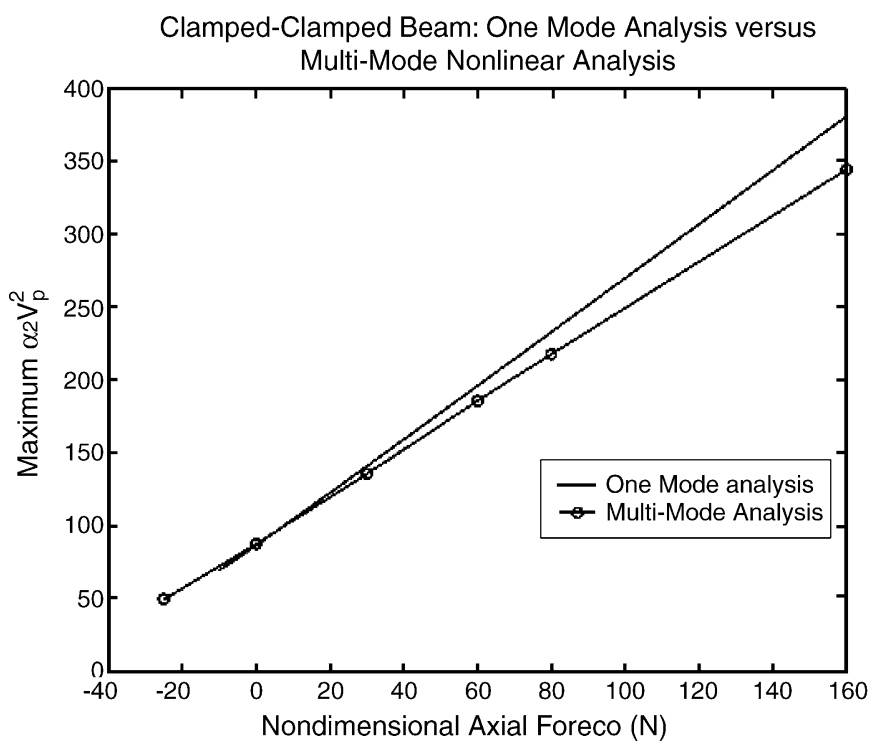

Fig. 6. Comparison of one-mode analysis and multi-mode analysis on pull-in $\alpha_{2} V_{\mathrm{p}}^{2}$ for clamped-clamped beam under different axial load $N$ s.
It is noticed that in both figures, the one-mode analysis only predicts the trend of critical maximum displacement. As the axial load $N$ increases and the difference between multi-mode and one-mode analysis is very significant. To explain this, the maximum beam deflection $(W(0.5))$ of the clamped-clamped beam is shown in Fig. 10 and the maximum of beam deflection $(W(1.0))$ of the cantilever beam is shown in Fig. 11. For both figures, the $x$-axis is $\alpha_{2} V_{\mathrm{p}}^{2}$, which increases from zero to the critical pull-in value. The critical pull-in points are marked by the circles. Those pull-in displacements in Figs. 8 and 9 are the same as those of Figs. 10 and 11. Clearly the deflections in both Figs. 10 and 11 show the similar behavior as $w_{1}$ shows in Fig. 2. The slopes of those curves dramatically change to a large number around the critical pull-in $\alpha_{2} V_{\mathrm{p}}^{2}$. At the critical pull-in $\alpha_{2} V_{\mathrm{p}}^{2}$, the slopes are infinite. So small change of $\alpha_{2} V_{\mathrm{p}}^{2}$ around the critical value can cause the huge change of the maximum beam displacements. Although the odd-mode method offers the

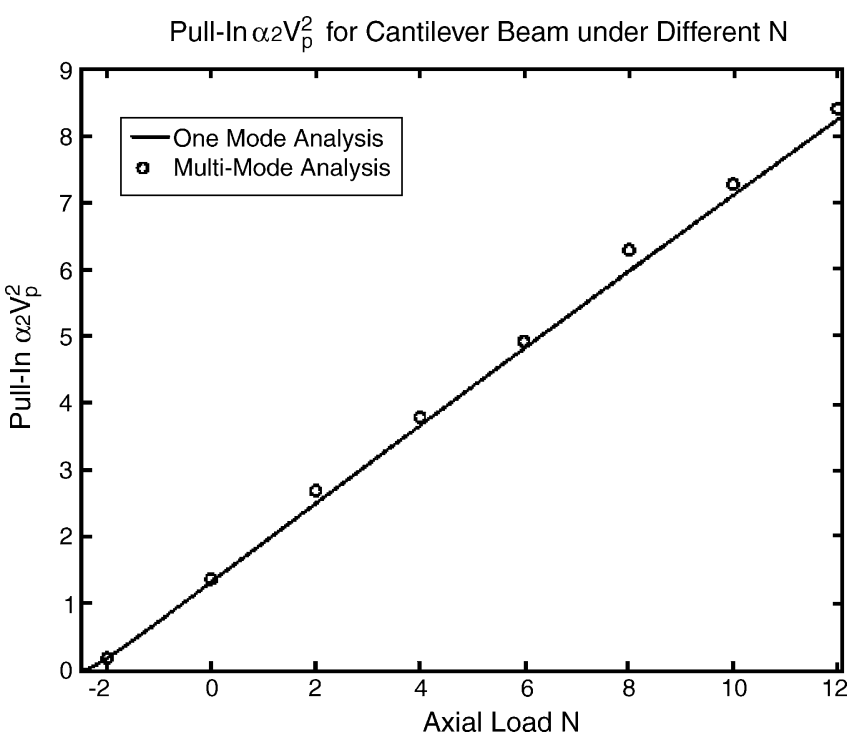

Fig. 7. Comparison of one-mode analysis and multi-mode analysis on pull-in $\alpha_{2} V_{\mathrm{p}}^{2}$ for cantilever beam under different axial load $N \mathrm{~s}$. 


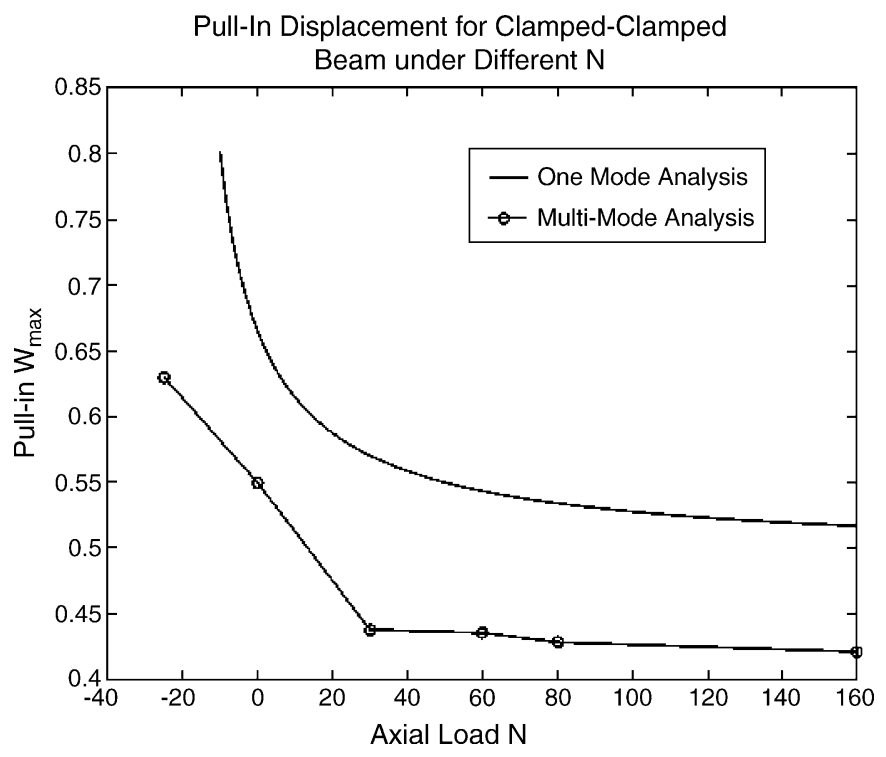

Fig. 8. Comparison of one-mode analysis and multi-mode analysis on pull-in $W_{\max }(0.5)$ for clamped-clamped beam under different axial load $N \mathrm{~s}$.

better convergence for the symmetric structure which we will discuss in the next section, the Galerkin computation method of multi-mode analysis still experiences the convergence difficulties around the pull-in $\alpha_{2} V_{\mathrm{p}}^{2}$. That is the reason why one-mode analysis offers much more accurate pull-in $\alpha_{2} V_{\mathrm{p}}^{2}$ than critical maximum displacements.

It is also noticed that in Fig. 8 the critical maximum displacements of the clamped-clamped beam $(W(0.5))$ decrease monotonically as the axial load $N$ increases. But in the Fig. 9, the critical maximum displacements of cantilever beam $(W(1.0))$ are the opposite. They increase monotonically as the axial load $N$ increases. To explain these two opposite trends, the weighted mechanical restoring force $F_{\mathrm{m}}$ and weighted electrical force $F_{\mathrm{e}}$

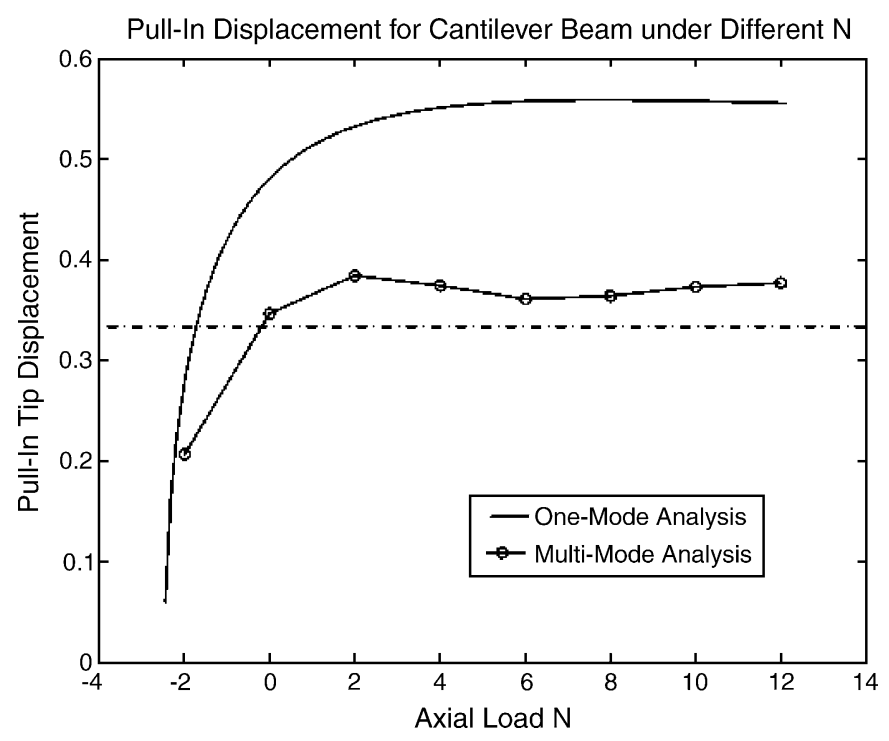

Fig. 9. Comparison of one-mode analysis and multi-mode analysis on pull-in $W_{\max }(1.0)$ for cantilever beam under different axial load $N \mathrm{~s}$.

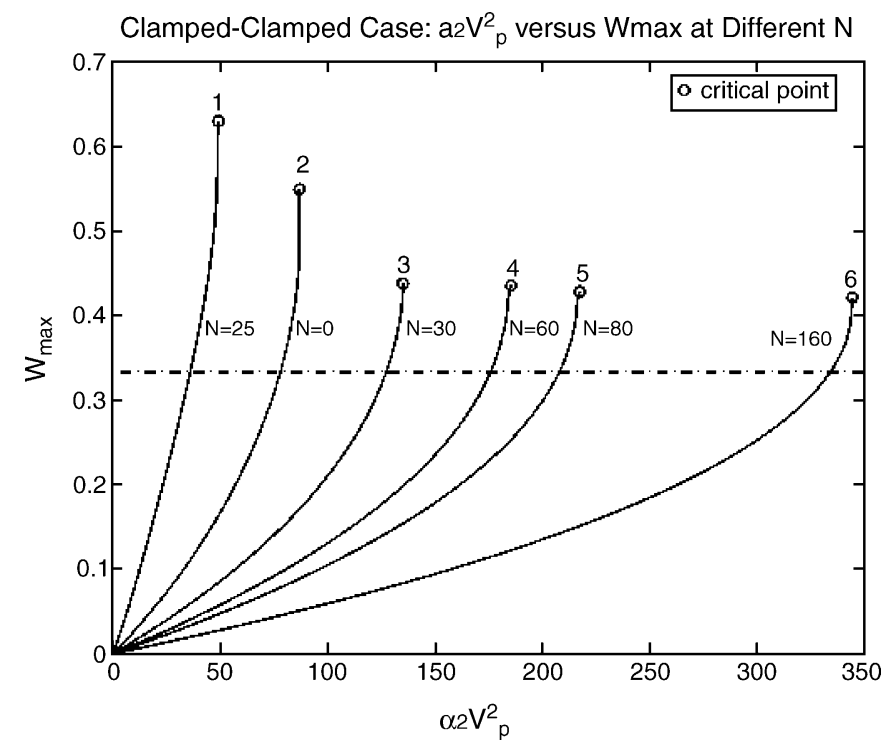

Fig. 10. $\alpha_{2} V_{\mathrm{p}}^{2}$ vs. $W_{\max }(0.5)$ for clamped-clamped beam under different axial load $N \mathrm{~s}$.

are introduced. $F_{\mathrm{m}}$ is defined as

$$
\begin{aligned}
F_{\mathrm{m}}= & \int_{0}^{1} \phi_{1} \frac{a_{1} \mathrm{~d}^{4} \phi_{1}}{\mathrm{~d} \xi^{4}} \mathrm{~d} \xi-\int_{0}^{1} \phi_{1} \\
& \times\left[\alpha_{1} \int_{0}^{1}\left(\frac{a_{1} \mathrm{~d} \phi_{1}}{\mathrm{~d} \xi}\right)^{2} \mathrm{~d} \xi+N\right] \frac{\mathrm{d}^{2}\left(a_{1} \phi_{1}\right)}{\mathrm{d} \xi^{2}} \mathrm{~d} \xi
\end{aligned}
$$

And $F_{\mathrm{e}}$ is defined as

$F_{\mathrm{e}}=\int_{0}^{1} \alpha_{2} V_{\mathrm{p}}^{2}\left(\phi_{1}+2 a_{1} \phi_{1}^{2}+3 a_{1}^{2} \phi_{1}^{3}+4 a_{1}^{3} \phi_{1}^{4}\right) \mathrm{d} \xi$

$\phi_{1}$ here plays two roles: mode shape and weight function.

As it can be clearly shown in Fig. 4, when the mechanical force curve is tangent to the electrical force curve, pull-in instability happens. In both Figs. 12 and 13, $\alpha_{1}$ is defined

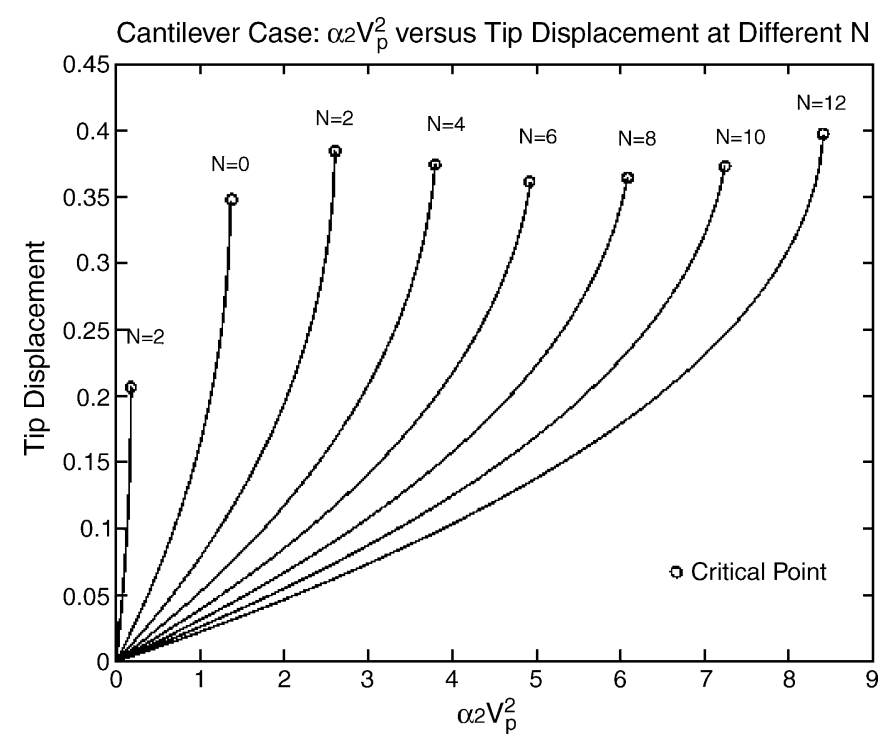

Fig. 11. $\alpha_{2} V_{\mathrm{p}}^{2}$ vs. $W_{\max }(1.0)$ for cantilever beam under different axial load Ns. 


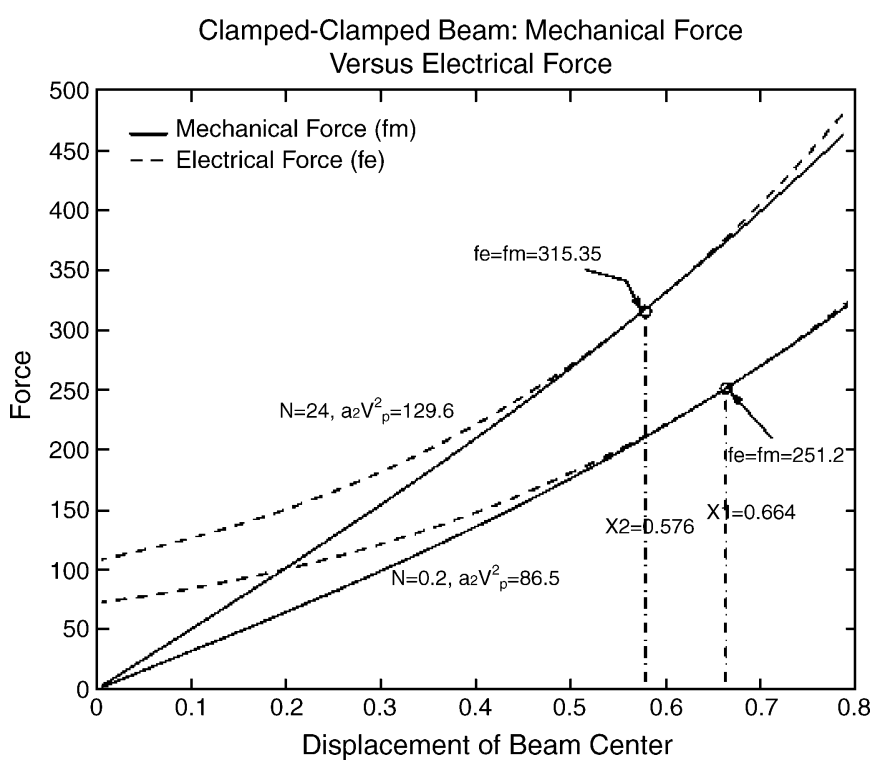

Fig. 12. The mechanical restoring force and electrical force curves of clamped-clamped beam at two pull-in states $(N=0.2$ and 24).

as 3.7. In Fig. 12, the $x$-axis is $a_{1} \phi_{1}(0.5)$ of the maximum clamped-clamped beam displacement at the center (here $\phi_{1}$ : the first mode shape of clamped-clamped beam). In Fig. 13, the $x$-axis is $a_{1} \phi_{1}(1.0)$ of the maximum cantilever beam displacement at the tip (here $\phi_{1}$ : the first mode shape of cantilever beam). In Fig. 12, there are two pull-in states for the clamped-clamped beam under $N=0.2$ and 24. Clearly, when $N$ increases from 0.2 to 24 , the beam stiffness increases, which is reflected by the slope increment of the mechanical force curve. At the same time, the pull-in $\alpha_{2} V_{\mathrm{p}}^{2}$ also increases, which is reflected by the moving-up of the electrical force curve. The exact same thing happens in Fig. 13 for cantilever beam. For clamped-clamped beam in Fig. 12, the tangent point, which indicates the critical maximum beam displacement, decreases from 0.664 to 0.576

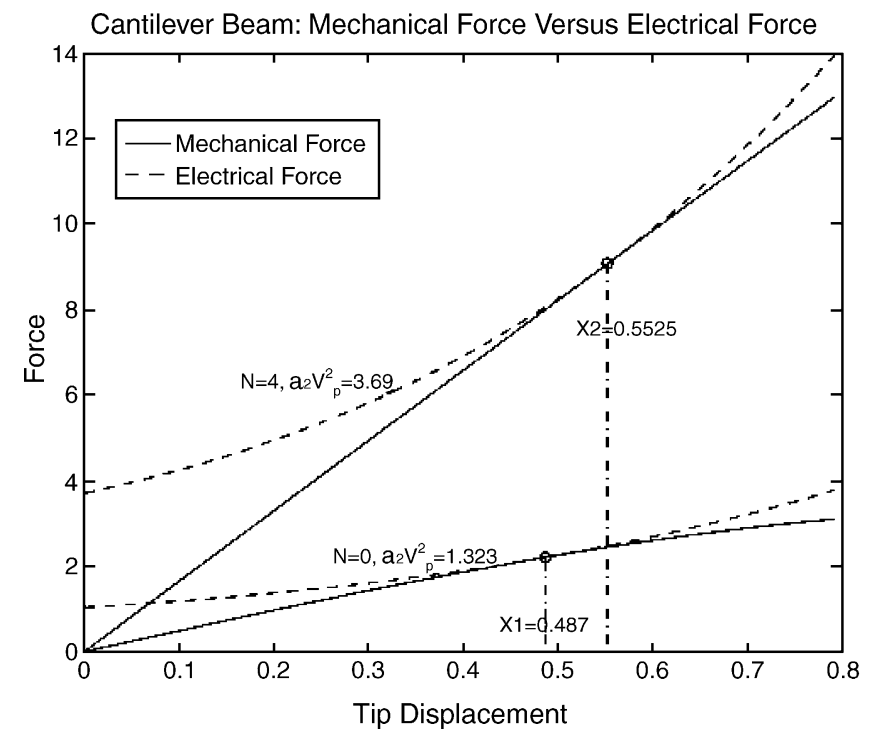

Fig. 13. The mechanical restoring force and electrical force curves of cantilever beam at two pull-in states $(N=0.0$ and 4$)$.

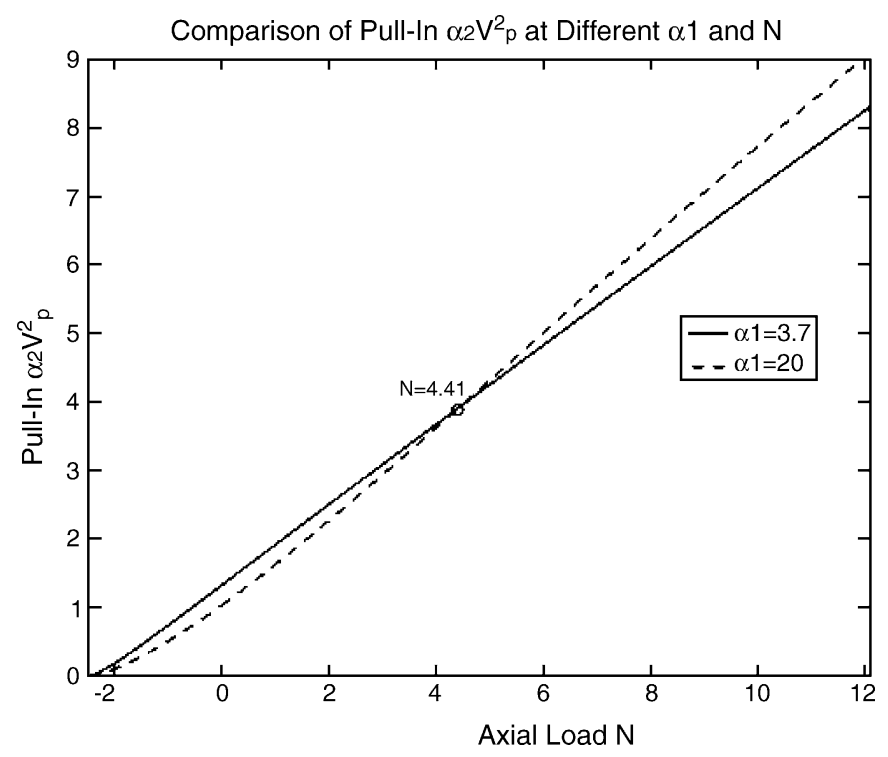

Fig. 14. Axial load $N$ vs. the pull-in $\alpha_{2} V_{\mathrm{p}}^{2}$ for cantilever beam. This figure shows the effect of $\alpha_{1}$ on the pull-in $\alpha_{2} V_{\mathrm{p}}^{2}$. The two curves have two different values of $\alpha_{1}=3.7$ and 20 .

as $N$ increases from 0.2 to 24 . However, for cantilever beam in Fig. 13, the tangent point increases from 0.487 to 0.5525 as $N$ increases from 0 to 4 . For both clamped-clamped and cantilever beams, as the axial load $N$ increases, both the beam stiffness and the pull-in $\alpha_{2} V_{\mathrm{p}}^{2}$ increase. As these two factors compete to each other, the $x$ coordinate of the tangent point (critical maximum beam displacement) may decrease (clamped-clamped beam) or increase (cantilever beam). So far, the parameter $\alpha_{1}$ is always defined as 3.7 for all the results shown above. In Eq. (8), the parameter $\alpha_{1}$ can be viewed as a parameter indicating the nonlinearity of a part of mechanical force. Small $\alpha_{1}$ indicates weak nonlinearity and big $\alpha_{1}$ indicates strong nonlinearity. In Fig. 14, the two curves with different $\alpha_{1}$ 's show how the pull-in $\alpha_{2} V_{p}^{2}$ changes with the axial load $N$. For cantilever beam, it is interesting to notice that the two curves cross each other at $N=4.41$. The results shown in Fig. 14 are done by one-mode analysis. For clamped-clamped beam, it is shown by Abdel-Rahman et al. [2] that the curves with different $\alpha_{1}$ never cross each other. It is also shown by Abdel-Rahman et al. that for clamped-clamped beam, bigger the $\alpha_{1}$ is, larger the pull-in $\alpha_{2} V_{p}^{2}$ is. This agrees with intuition because when only $\alpha_{1}$ increases, it means the nonlinear part of the mechanical force increases. As the result, the whole beam mechanical restoring force is expected to increase. So the bigger pull-in $\alpha_{2} V_{\mathrm{p}}^{2}$ is expected. But as Eqs. (12)-(14) indicate that the change of $\alpha_{1}$ will change every coefficient of Eq. (12). Although the mechanical restoring force increases due to the increment of $\alpha_{1}$, the critical maximum beam displacement also changes. Fig. 15 shows the critical maximum beam displacement $(W(1.0))$ of the cantilever beam as the axial load $N$ changes. It is worthy noticing that the two curves also cross each other at $N=4.41$. So for cantilever beam, although bigger $\alpha_{1}$ means the bigger beam nonlinearity of mechanical force and more restoring mechanical force, the required critical maximum displacement with bigger $\alpha_{1}$ is also smaller until $N=4.41$. The smaller critical maximum displacement requires the less pull-in $\alpha_{2} V_{\mathrm{p}}^{2}$ to let the system 
Comparison of Pull-In Displacement at Different $\alpha 1$ and $\mathrm{N}$

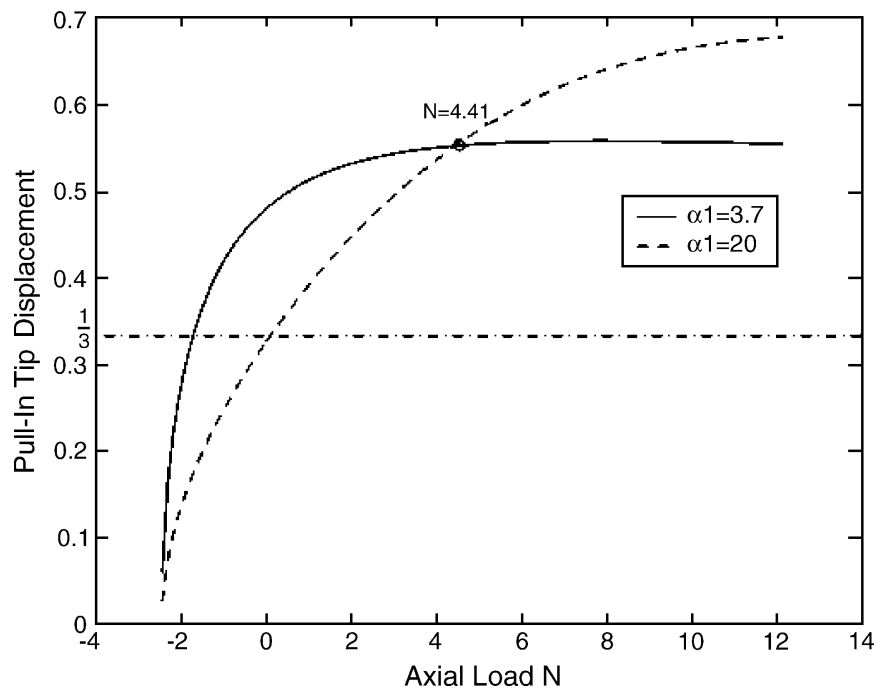

Fig. 15. Axial load $N$ vs. the pull-in maximum displacement $W_{\max }(1.0)$ for cantilever beam. This figure shows the effect of $\alpha_{1}$ on the pull-in maximum displacement $W_{\max }(1.0)$. The two curves have two different $\alpha_{1}=3.7$ and 20 .

to reach the pull-in position. But as the system gets stiffer due to the increment of parameter $\alpha_{1}$, its restoring force increases. These two factors compete to each other, as the result, cantilever beam with bigger $\alpha_{1}$ actually has smaller pull-in $\alpha_{2} V_{\mathrm{p}}^{2}$ shown in Fig. 15 until $N=4.41$.

\section{Comparison of all-mode method (AMM) and odd-mode method (OMM) for clamped-clamped beam}

For clamped-clamped beam case, if $W$ is expanded as the following odd-mode only series, the computation results are better than those of all-mode expansion.

$W=\sum_{i=1,2,3 \ldots}^{M} a_{2 i-1} \phi_{2 i-1}(\xi)$

Odd modes are symmetric modes and even modes are the antisymmetric modes. For clamped-clamped beam, the boundary conditions are symmetric. From the governing equation of (8) for clamped-clamped beam, it can also be shown that the through the whole domain, the electrical force is uniformly distributed. One of odd-mode method advantages is obvious. It dramatically reduces the mode number required to have the same precision as all-mode method expansion. It also reduces the numerical fluctuation around the pull-in instability area. The fluctuation is due to the slope of voltage-displacement curve increases to infinity at the pull-in point. And the numerical computation on the pullin displacement is extremely sensitive to the step size. For the sake of brevity, we only show this sensitivity in the computation of plate in the next section. In contrast, for the analytical solution of one-mode analysis, there is no such problem.

The numerical fluctuation around the pull-in is shown in Fig. 16. Fig. 16 shows the comparison of AMM and OMM at $\alpha_{1}=3.7$ and $N=30$. There are five modes $\phi_{1}, \phi_{2}, \phi_{3}, \phi_{4}, \phi_{5}$ used in AMM and three modes $\phi_{1}, \phi_{3}, \phi_{5}$ used in OMM. The

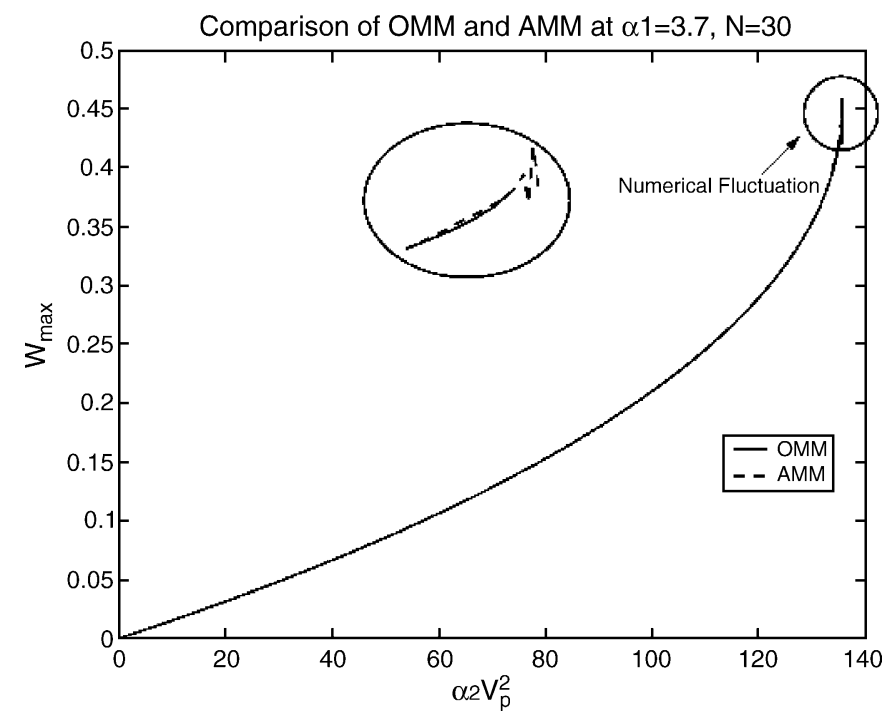

Fig. 16. Comparison of $\alpha_{2} V_{\mathrm{p}}^{2}$ vs. $W_{\max }$ computed by OMM and AMM at $N=30$, $\alpha_{1}=3.7$.

results are almost exactly the same except those in the tiny area of $135.3<\alpha_{2} V_{\mathrm{p}}^{2}<135.8$, which is very close to the pull-in. There is obvious numerical fluctuation in AMM and it happens to be no numerical fluctuation in this case for OMM. Generally speaking, OMM has much less numerical fluctuation than AMM.

\section{Continuous system: plate with four edges simply-supported}

\subsection{Plate governing equation and nondimensionalization}

For the pull-in study on the plate, here only the case of the plate with four edges simply-supported is selected for the simplicity reason. The schematic diagram of the plate and its coordinate are shown in Fig. 17. The governing equation for the

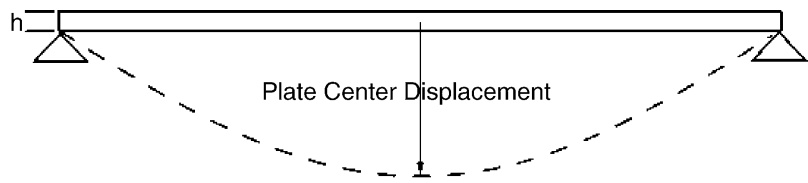

Stationary Electrode

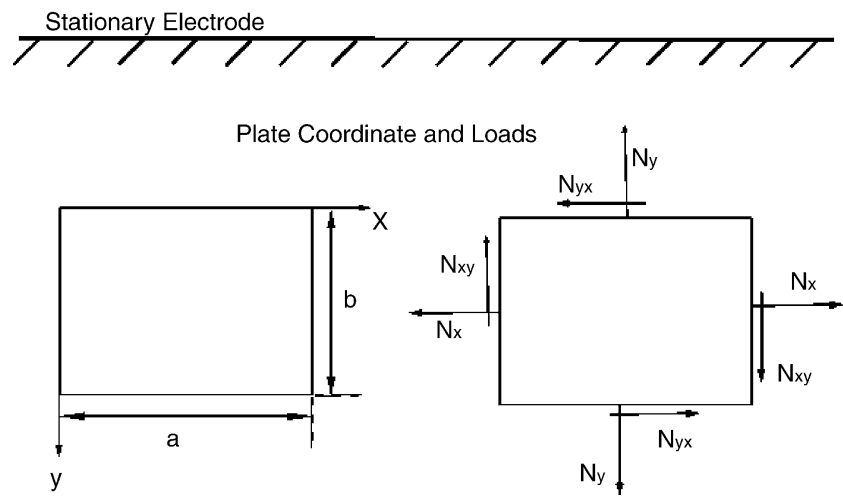

Fig. 17. Schematics of plate coordinate and load scenario. 
plate under axial and transverse loads is given as [28]

$$
\begin{aligned}
& \frac{\partial^{4} w}{\partial x^{4}}+2 \frac{\partial^{4} w}{\partial x^{2} \partial y^{2}}+\frac{\partial^{4} w}{\partial y^{4}} \\
& \quad=\frac{1}{D}\left(q+N_{x} \frac{\partial^{2} w}{\partial x^{2}}+N_{y} \frac{\partial^{2} w}{\partial y^{2}}+2 N_{x y} \frac{\partial^{2} w}{\partial x \partial y}\right)
\end{aligned}
$$

$w=w(x, y)$ is the plate transverse deflection. $D$ is flexural rigidity of the plate and is given as $D=E h^{3} / 12\left(1-\mu^{2}\right)$, here $E$ is Young's modulus, $h$ the plate thickness and $\mu$ is Poisson's ratio. $N_{x}, N_{y}$ and $N_{x y}$ are the loads per unit length of different directions shown in Fig. 17. $N_{x}$ and $N_{y}$ are normal loadings. $N_{x y}$ is shear loading. $q=q(x, y)$ is the transverse load intensity (load per unit area). Here only the electrical loading contributes to this load intensity. Therefore, the load intensity is given as $q=\varepsilon V^{2} / 2(d-w)^{2}[1] . \varepsilon, d$ and $V$ are defined the same as those for one DOF system and beams above.

In order to nondimensionalize Eq. (21), the following dimensionless numbers are introduced

$W=\frac{w}{d}, \quad \xi=\frac{x}{a}, \quad \eta=\frac{y}{b}$

$a$ and $b$ are the plate length and width shown in Fig. 17 .

Eq. (21) now is nondimensionalized as

$$
\begin{aligned}
& \frac{\partial^{4} W}{\partial \xi^{4}}+\frac{2}{\psi^{2}} \frac{\partial^{4} W}{\partial \xi^{2} \partial \eta^{2}}+\frac{\partial^{4} W}{\psi^{4} \partial \eta^{4}} \\
& \quad=\frac{a_{1}}{(1-W)^{2}}+a_{2} \frac{\partial^{2} W}{\partial \xi^{2}}+a_{3} \frac{\partial^{2} W}{\partial \eta^{2}}+a_{4} \frac{\partial^{2} W}{\partial \xi \partial \eta}
\end{aligned}
$$

The dimensionless deflection $W$ here is assumed to have only one-mode shape expansion, which is $W=b_{11} \phi_{11}(\xi, \eta)$. $b_{11}$ is the modal amplitude to be determined and $\phi_{11}$ is the plate mode shape with the lowest eigenfrequency. For rectangular plates with different boundary conditions, the mode shapes with different eigenfrequencies are given by Gorman [29]. For the plate with four edges simply-supported, the expansion can be simply expressed as $W=b_{11} \sin (\pi \xi) \sin (\pi \eta)$ Substitute this expression into Eq. (25), times $\phi_{11}$ and integrate in the $[01 ; 01]$ domain, now the governing equation changes as

$$
\begin{aligned}
b_{11} \int_{0}^{1} \int_{0}^{1} \phi_{11}\left(\frac{\partial^{4} \phi_{11}}{\partial \xi^{4}}+\frac{2}{\psi^{2}} \frac{\partial^{4} \phi_{11}}{\partial \xi^{2} \partial \eta^{2}}+\frac{\partial^{4} \phi_{11}}{\psi^{4} \partial \eta^{4}}\right) \mathrm{d} \xi \mathrm{d} \eta \\
=a_{1} \int_{0}^{1} \int_{0}^{1} \phi_{11}\left[1+2 b_{11} \phi_{11}+3\left(b_{11} \phi_{11}\right)^{2}\right. \\
\left.\quad+4\left(b_{11} \phi_{11}\right)^{3}\right] \mathrm{d} \xi \mathrm{d} \eta+\int_{0}^{1} \int_{0}^{1} b_{11} \phi_{11} \\
\quad \times\left(a_{2} \frac{\partial^{2} \phi_{11}}{\partial \xi^{2}}+a_{3} \frac{\partial^{2} \phi_{11}}{\partial \eta^{2}}+a_{4} \frac{\partial^{2} \phi_{11}}{\partial \xi \partial \eta}\right) \mathrm{d} \xi \mathrm{d} \eta
\end{aligned}
$$

After the integration, the equation above can be written as a cubic equation of $b_{11}$ as follows

$b_{11}^{3}+\kappa_{1} b_{11}^{2}+\kappa_{2} b_{11}+\kappa_{3}=0$

Here $\kappa_{1}, \kappa_{2}$ and $\kappa_{3}$ are defined as

$$
\begin{aligned}
& \int_{0}^{1} \int_{0}^{1} \phi_{11}\left(2 a_{1} \phi_{11}+a_{2}\left(\left(\partial^{2} \phi_{11}\right) /\left(\partial \xi^{2}\right)\right)+a_{3}\left(\left(\partial^{2} \phi_{11}\right) /\left(\partial \eta^{2}\right)\right)+a_{4}\left(\left(\partial^{2} \phi_{11}\right) /(\partial \xi \partial \eta)\right)\right. \\
& \kappa_{2}=\frac{\left.-\left(\left(\partial^{4} \phi_{11}\right) /\left(\partial \xi^{4}\right)\right)-\left(2 / \psi^{2}\right)\left(\left(\partial^{4} \phi_{11}\right) /\left(\partial \xi^{2} \partial \eta^{2}\right)\right)-\left(\left(\partial^{4} \phi_{11}\right) /\left(\psi^{4} \partial \eta^{4}\right)\right)\right) \mathrm{d} \xi \mathrm{d} \eta}{J} \\
& \kappa_{1}=\frac{3 a_{1} \int_{0}^{1} \int_{0}^{1} \phi_{11}^{3} \mathrm{~d} \xi \mathrm{d} \eta}{J}, \quad \kappa_{3}=\frac{a_{1} \int_{0}^{1} \int_{0}^{1} \phi_{11} \mathrm{~d} \xi \mathrm{d} \eta}{J}
\end{aligned}
$$

Here $\psi$ is the aspect ratio defined as $\psi=b / a . a_{1}, a_{2}, a_{3}$ and $a_{4}$ are defined as follows

$a_{1}=\frac{a^{4} V^{2}}{2 D d^{3}}, \quad a_{2}=\frac{N_{x} a^{2}}{D}, \quad a_{3}=\frac{N_{y} a^{2}}{\psi^{2} D}, \quad a_{4}=\frac{2 N_{x y} a^{2}}{\psi D}$

\subsection{One-mode analysis of plate}

The one-mode analysis is very similar to those of beams. The electrical load intensity part $q(x, y)$ is expanded into Taylor series, therefore, Eq. (23) is changed as the following

$$
\begin{aligned}
& \frac{\partial^{4} W}{\partial \xi^{4}}+\frac{2}{\psi^{2}} \frac{\partial^{4} W}{\partial \xi^{2} \partial \eta^{2}}+\frac{\partial^{4} W}{\psi^{4} \partial \eta^{4}} \\
& =a_{1}\left(1+2 W+3 W^{2}+4 W^{3}\right)+a_{2} \frac{\partial^{2} W}{\partial \xi^{2}} \\
& +a_{3} \frac{\partial^{2} W}{\partial \eta^{2}}+a_{4} \frac{\partial^{2} W}{\partial \xi \partial \eta}
\end{aligned}
$$

$J$ here is defined as $J=4 a_{1} \int_{0}^{1} \int_{0}^{1} \phi_{11}^{4} \mathrm{~d} \xi \mathrm{d} \eta$.

Very similarly as the Cardan solution used in one DOF system and beams cases, now $Q$ is defined as

$$
\begin{aligned}
& Q=\left(\frac{p}{3}\right)^{3}+\left(\frac{q}{2}\right)^{2} \\
& \left(p=-\frac{\kappa_{1}^{2}}{3}+\kappa_{2}, \quad q=2\left(\frac{\kappa_{1}}{3}\right)^{3}-\left(\frac{\kappa_{1} \kappa_{2}}{3}\right)+\kappa_{3}\right)
\end{aligned}
$$

$\kappa_{1}, \kappa_{2}$ and $\kappa_{3}$ contain $a_{1}$. The pull-in point can be determined from equation $Q=\left(a_{1}\right)=0$ as $Q$ defined in Eq. (29). Thus, $a_{1}$ can be calculated from the equation $Q\left(a_{1}\right)=0$. Once $a_{1}$ is determined, the pull-in displacement can also be determined from Cardan solution. Therefore, the one-mode analysis basically is to solve the cubic equation of the modal amplitude of the plate mode shape with the lowest eigenfrequency. The onemode analysis does not assume the effective stiffness to treat the continuous system as the one DOF system. The discretization, which assumes only one-mode shape of plate deflection, and Taylor series expansion together help to generate this cubic 
1st Mode Shape

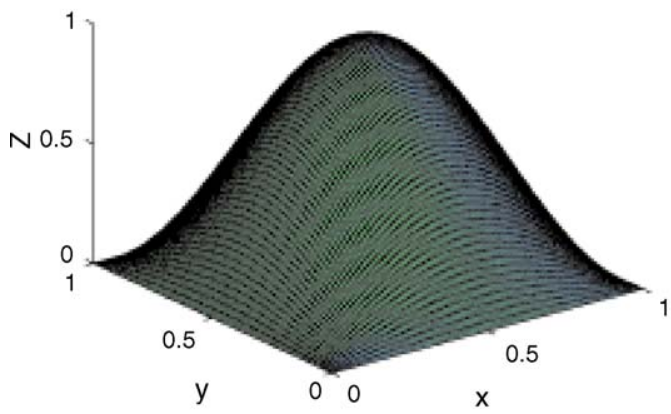

3rd Mode Shape

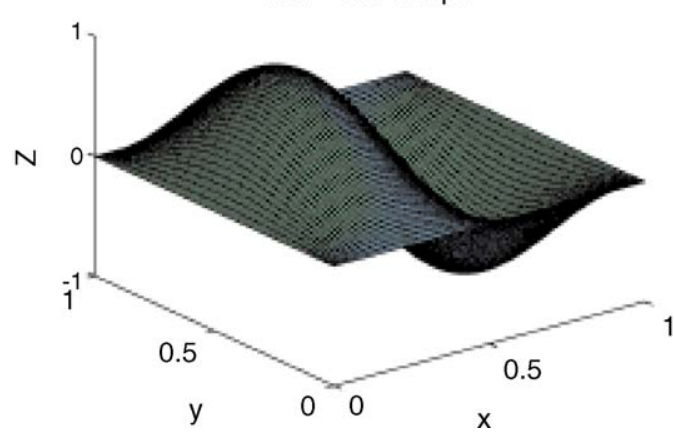

2nd Mode Shape

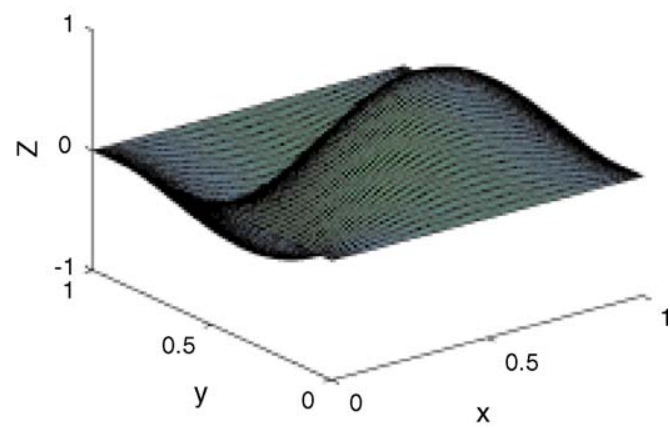

4rd Mode Shape

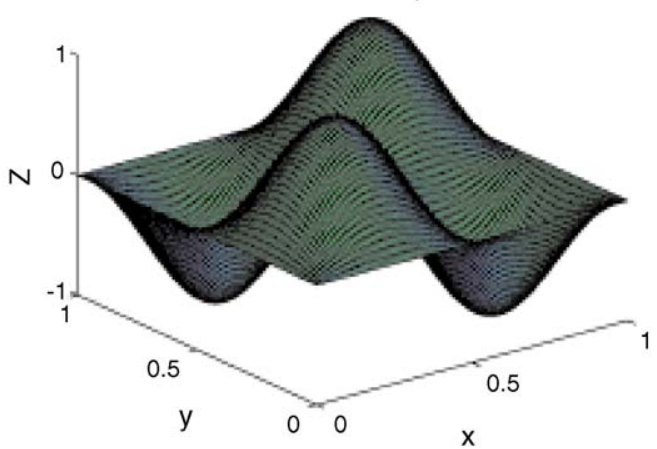

Fig. 18. The first four lowest four mode shapes used in the numerical multi-mode analysis computation.

equation for the continuous system. By setting $Q\left(a_{1}\right)=0$, this cubic equation can directly solve the system pull-in $a_{1}$, thus the voltage and displacement. Although this one-mode analysis in practice requires some numerical methods to deal with the integration and root solving, fundamentally it is a cubic equation with the available analytical solution.

\subsection{Multi-mode analysis of plate}

Once again, for the numerical multi-mode analysis, it is unnecessary to have a Taylor series expansion for the electrical loading part. The plate is assumed to have such expansion

$W=\sum_{i=1,2,3 \ldots}^{M_{1}} \sum_{j=1,2,3 \ldots}^{M_{2}} b_{i j} \phi_{i j}(\xi, \eta)$

Here $M_{1}$ and $M_{2}$ are mode numbers. $M_{1}$ and $M_{2}$ may not necessarily equal each other. $b_{i j}$ is the modal amplitude to be determined. $\phi_{i j}$ is the plate mode shape. The four mode shapes with the lowest four eigenfrequencies are shown in Fig. 18. They are 1-1, 1-2, 2-1, 2-2 types of the mode shapes. Substitute the expression above into Eq. (23) and use the same Galerkin method shown in one-mode analysis on the plate above. For $M_{1} \times M_{2}$ modal expansion, there are $M_{1} \times M_{2}$ equations. Newton-Rhapson method is applied to the nonlinear equation set. It is also noticed that for the four modes, only 1-1 and 2-2 are symmetric among all of these four mode shapes. The discussion on the AMM and OMM for clamped-clamped beam case is valid for the plate studied here as its boundary conditions (four edges simply-supported) and electrical load are symmetric. Only these two modes can be useful during the computation.

\section{Results and discussion for the pull-in instability of the plate}

Fig. 19 shows the pull-in $a_{1}$ calculated by the one-mode analysis method. The plate for all the cases computed here is a square plate, which means the aspect ratio $\psi=1$. Here $a_{4}$ is set to be zero, which is no shear loading case. $\beta$ is set as $\beta=a_{3} / a_{2}$, which indicates the relation of the axial loads in $x$ and $y$ directions. The pull-in $a_{1}$ versus $a_{2}$ for three sets of different $\beta$ s are shown in Fig. 19. As it is shown, the pull-in $a_{1}$ increases as the axial loading increases. When the axial loading increases, the plate becomes stiffer. The plate is stiffened by the axial loadings of both directions. As the result, larger voltage is required to have the system to pull-in.

As we mention above that the multi-mode computation of pull-in displacement is largely dependent on the step size of $a_{1}$. Because the slope of $a_{1}$ versus displacement curve becomes very large and approaches infinity around the pull-in point, any tiny change of $a_{1}$ can cause very large change of pull-in displacement. Fig. 20 shows this pull-in computation sensitivity to step size of $a_{1}$. In Fig. 20, the plate center deflection $(W(1 / 2,1 / 2))$ increases as the $a_{1}$ gradually changes from zero to its pull-in value. The three computation results of plate center pull-in displacement under different $a_{1}$ step sizes of $0.2,0.3$ and 0.4 are 


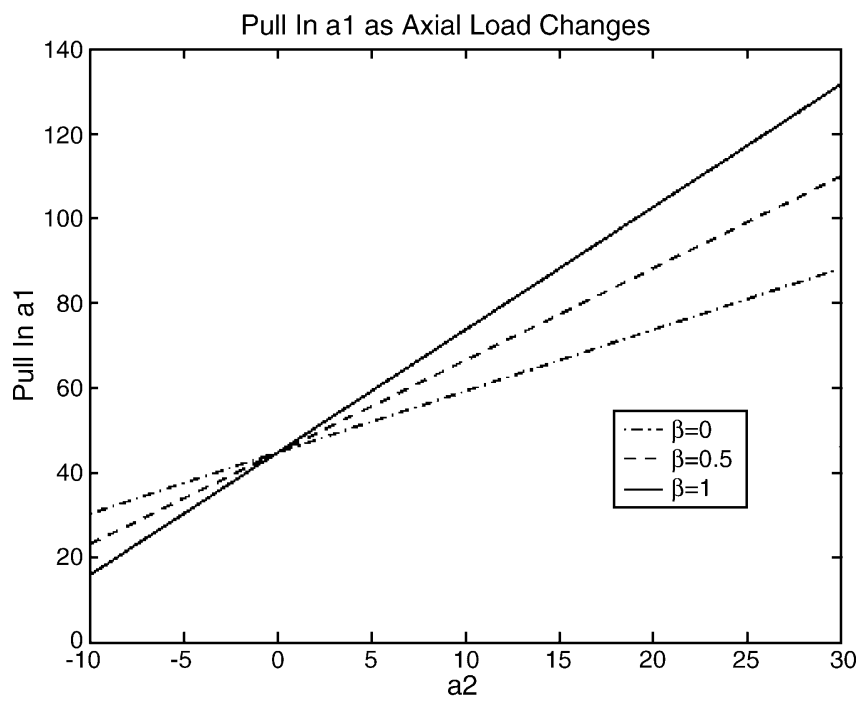

Fig. 19. Different pull-in $a_{1}$ for different load cases calculated by one-mode analysis.

shown in Fig. 20. The plate center pull-in displacement is computed as $0.5142,0.479$ and 0.6172 , respectively. For one-mode analysis, this value is calculated as 0.5827 . In Fig. 20 case, the plate is under no axial loading in both directions. While, the pull-in $a_{1}$ is not affected by this step size very much. The pull-in $a_{1}$ computed by the three different step sizes is $41.2,41.4$ and 41.6, respectively. For the multi-mode analysis here, increasing mode number does not help to improve the computation accuracy much because the first mode shape is the predominant deflection shape. However, it may affect the convergence around the pull-in point.

Fig. 21 shows the plate center deflection changes from $a_{1}=0$ to its pull-in value for the different $x$-direction axial load cases $\left(a_{2}\right)$. The other load related parameters $a_{3}$ and $a_{4}$ are both set to be zero. The step size of $a_{1}$ is set to be 0.2 for all the cases shown in Fig. 21. As the axial load $\left(a_{2}\right)$ increases, the pull-

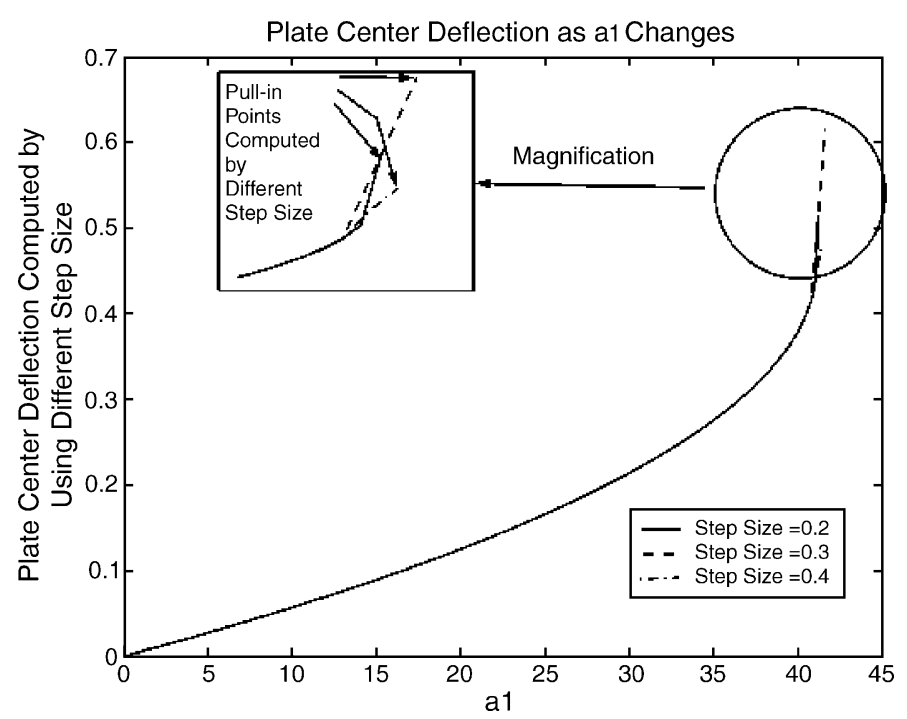

Fig. 20. Step size influence on the computation of the numerical multi-mode analysis.

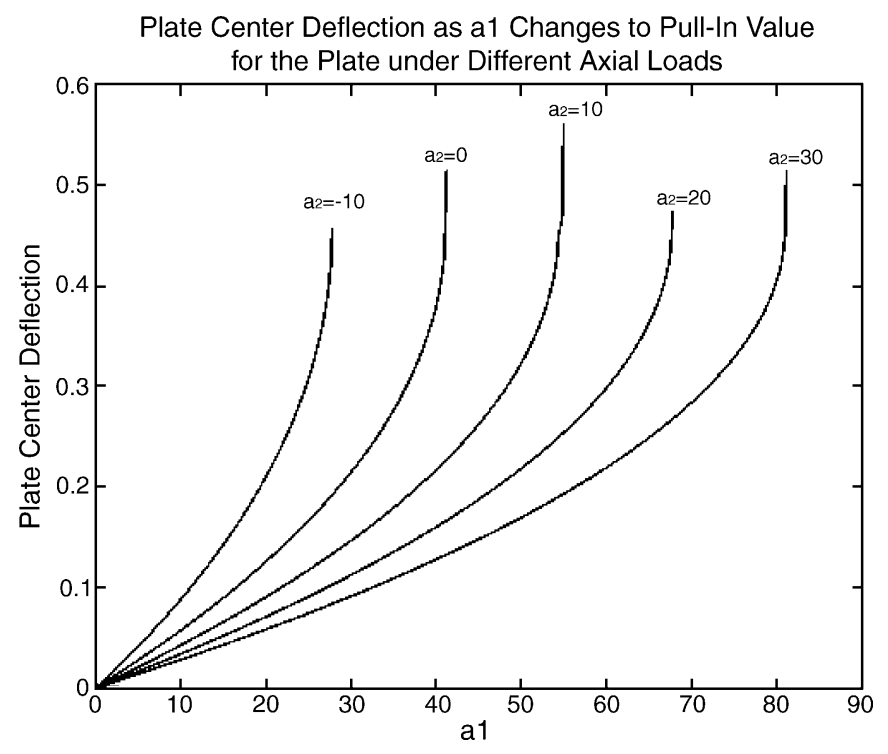

Fig. 21. The plate center deflection as $a_{1}$ gradually approaching its pull-in value for different load cases.

in $a_{1}$ also increases. For the plate center pull-in displacement, it is irregular. The similar irregularity property is also shown in the computation results of the pull-in center displacement of the clamped-clamped beam by Abdel-Rahman et al. [2]. Fig. 22 shows the comparison of the pull-in $a_{1}$ computed by one-mode and multi-mode analysis method. $a_{3}$ and $a_{4}$ are also set zero in this case. As it is shown, the pull-in $a_{1}$ increases linearly as $a_{2}$ increases. This phenomenon is also shown in the clamped-clamped beam structure under axial loading we show above and the study of Abdel-Rahman et al. [2]. As it is shown in Fig. 19, this linear relation still keeps under both $x$ and $y$ directional axial loadings. The multi-mode analysis pull-in $a_{1}$ value is always smaller than the one-mode analysis result. And as the axial load $\left(a_{2}\right)$ increases, the computation difference of

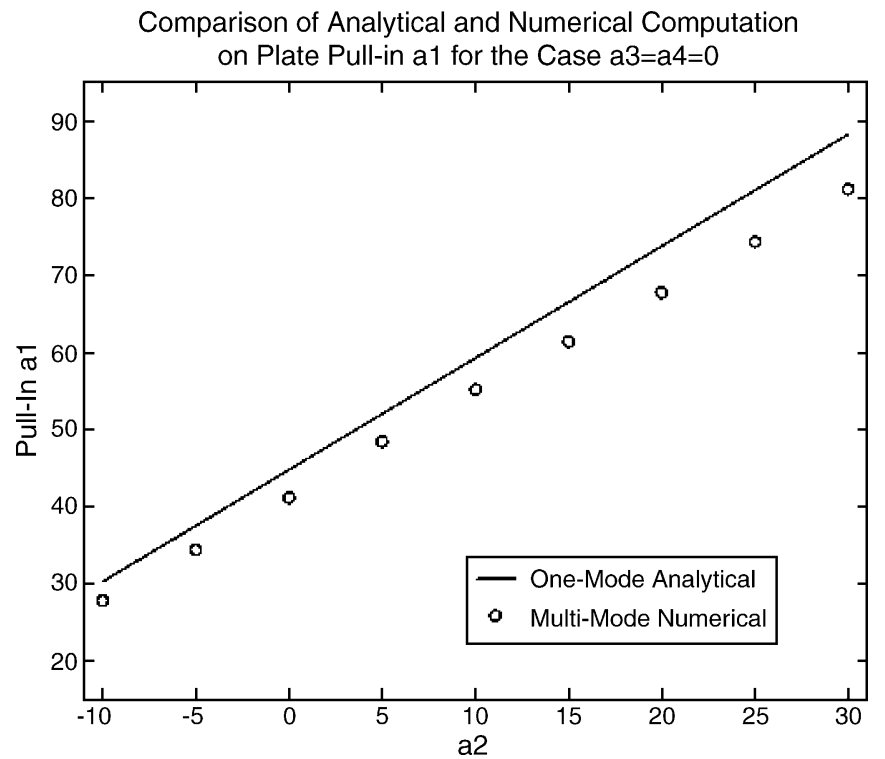

Fig. 22. Comparison of the pull-in $a_{1}$ computed by the analytical one-mode analysis and numerical multi-mode analysis. 


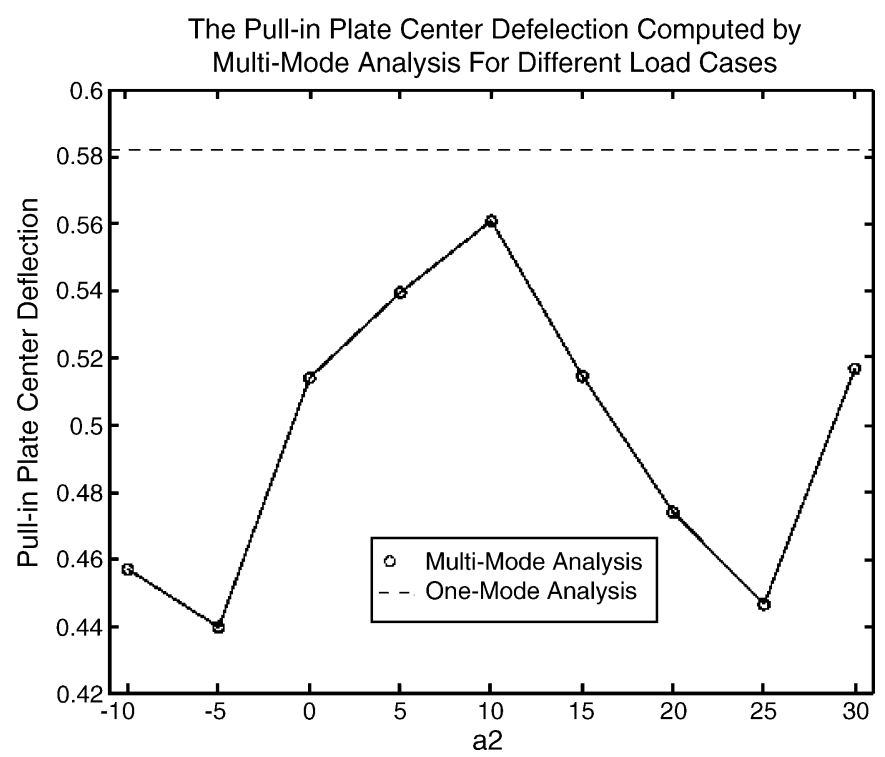

Fig. 23. Comparison of the plate center pull-in displacement computed by the analytical one-mode analysis and numerical multi-mode analysis.

the two methods enlarges. Although the one-mode analysis is to use the analytical solution of the cubic equation and the multimode analysis is to use Newton-Rhapson methods, the solution method is not the main reason causing the difference. Fig. 23 shows the plate center pull-in displacement for different axial $a_{2}$ cases. $a_{3}, a_{4}$ are also both set to be zero. For the one-mode analysis, the plate center pull-in displacement keeps constant as 0.5827 for different loading cases. While for the multi-mode analysis, it is irregular pattern. The $a_{1}$ step size used in the computation is also 0.2 .

\section{Summary and conclusions}

The pull-in $\alpha_{2} V_{\mathrm{p}}^{2}$ and critical maximum displacements of clamped-clamped and cantilever beam are compared. For both clamped-clamped and cantilever beams, the pullin $\alpha_{2} V_{\mathrm{p}}^{2} \mathrm{~s}$ increase as axial load $N$ increases. But when axial load $N$ increases, the critical maximum displacement of clamped-clamped beam $W(0.5)$ monotonically decreases but the critical maximum displacement of cantilever beam $W(1.0)$ monotonically increases. Although increasing axial load $N$ increases beam mechanical stiffness and the pull-in $\alpha_{2} V_{\mathrm{p}}^{2}$, it will not necessarily improve the critical maximum beam displacement. In other words, the tuning range of voltage can be definitely improved by increasing axial load. But the tuning range of critical maximum displacements will not necessarily be improved by doing the same thing. As a structure in MEMS actuators, switcher, etc. under electrical actuation, the tuning displacement range of clamped-clamped beam will decrease as the axial load $N$ increases. Cantilever beam has much smaller stiffness, but both the tuning voltage and displacement will increase as the axial load $N$ increases.

One-mode analysis method shows its accurate prediction on the pull-in $\alpha_{2} V_{\mathrm{p}}^{2}$. One-mode analysis given in this paper basically is to use Taylor series expansion and Galerkin method to transform the nonlinear differential equation into a cubic equation. The validity of one-mode analysis relies on the fact that the first mode is the dominant deflection shape for the beam/plate structure under electrical actuation and small axial load. The advantages of one-mode analysis are the analytical solution available and only small amount of computation needed. For one-mode analysis, it should also be aware that the truncation error due to Taylor series expansion may be large when axial loading or structural deflection is large. Multi-mode analysis is a more general analysis tool. It does not need to use Taylor series to approximate the electrical force. In that sense, it is more accurate than one-mode analysis. It uses Newton-Rhapson method to solve the equation and pays the price of much more computation effort than one-mode analysis. Multi-mode and one-mode analysis are two quite different methods, which as a result, can be used to verify each other.

As mentioned above, the first mode shape is the dominant beam/plate deflection shape. Mode number is not a major factor influencing the computation results of the multi-mode analysis. The error sources of one-mode analysis are the truncation error due to the Taylor series expansion on the electrical force part and the possible participation of higher mode shapes into the deflection. As the axial load increases, the pull-in voltage increases. The pull-in voltage behaves like a magnification factor which enlarges the difference between the two analysis results when the axial loading increases. Numerically we use the property of the slope of voltage-displacement approaching infinity to determine the pull-in instability, the computational results of multi-mode analysis is largely dependent on the voltages step size. Therefore, the pull-in displacement computed by multi-mode analysis can also problematic when using the inappropriate voltage step sizes. In practice, the control parameter is the voltage and the pull-in voltages computed by one-mode analysis and multi-mode analysis agree well with each other for the range of small axial loading (both compressive and tensile).

\section{Acknowledgments}

Zhang is thankful to the financial supports from both the National Natural Science Foundation of China (NSFC, Grant no. 10502050) and the Scientific Research Foundation for the Returned Overseas Chinese Scholars, State Education Ministry. Zhao is supported by the Distinguished Young Scholar Fund of National Natural Science Foundation of China (NSFC, Grant no. 10225209), the key project from Chinese Academy of Sciences (Grant no. KJCX-SW-L2) and NSFC project (Grant no. 90305020).

\section{Appendix A}

The linear dynamic governing equation for beam is

$E I \frac{\partial^{4} y}{\partial x^{4}}-p \frac{\partial^{2} y}{\partial x^{2}}+m \frac{\partial^{2} y}{\partial t^{2}}=0$

$y$ is the beam transverse displacement, $m$ the mass per unit length of the beam, $p$ the axial load, when $p>0$, it is tension and when $p<0$, it is compression. 
The beam length is $l$. Assume $y$ has such solution form, $y=V(x) \mathrm{e}^{i \omega t}$. Substitute this solution form into Eq. (31) and introduce nondimensional parameter $\xi=x / l$. The governing Eq. (31) becomes

$-m \omega^{2} l^{4} V+E I V^{\prime \prime \prime \prime}-p l^{2} V^{\prime \prime}=0$

Here ()$^{\prime}=\partial / \partial \xi$. Let $k^{2}=\left|\frac{p l^{2}}{E I}\right|$ and $\beta=m \omega^{2} l^{4} / E I$. For tension case $(p>0)$, the governing equation is

$V^{\prime \prime \prime \prime}-k^{2} V^{\prime \prime}-\beta^{4} V=0$

For compression case $(p<0)$, the governing equation is

$V^{\prime \prime \prime \prime}+k^{2} V^{\prime \prime}-\beta^{4} V=0$

For tension case, the solution of $V$ is

$V=A \mathrm{e}^{f_{2} \xi}+B \mathrm{e}^{-f_{2} \xi}+C \sin \left(f_{1} \xi\right)+D \cos \left(f_{1} \xi\right)$

$A, B, C, D$ are four unknown constants. $f_{1}$ and $f_{2}$ are defined as $f_{1}=\sqrt{\beta_{1}-\left(k^{2} / 2\right)}$ and $f_{2}=\sqrt{\beta_{1}+\left(k^{2} / 2\right)}$. Here $\beta_{1}=$ $\sqrt{\beta^{4}+\left(k^{4} / 4\right)}$. The four dimenisonless boundary conditions are given as

$$
\begin{aligned}
& V(0)=0, \quad \frac{\partial V(0)}{\partial \xi}=0 ; \quad \frac{\partial^{2} V(1)}{\partial \xi^{2}}=0, \\
& \frac{\partial^{3} V(1)}{\partial \xi^{3}}-k^{2} \frac{\partial V(1)}{\partial \xi}=0
\end{aligned}
$$

Substitute the $V$ solution form into these four boundary conditions, then there is a $4 \times 4$ determinant. Solving the determinant will give the eigenfrequencies of the systems and by substituting each individual eigenfrequency into that $4 \times 4$ matrix, the mode shape can be found. For compression case, the solution has the form

$V=A \mathrm{e}^{f_{1} \xi}+B \mathrm{e}^{-f_{1} \xi}+C \sin \left(f_{2} \xi\right)+D \cos \left(f_{2} \xi\right)$

And the boundary conditions change as

$$
\begin{aligned}
& V(0)=0, \quad \frac{\partial V(0)}{\partial \xi}=0 ; \quad \frac{\partial^{2} V(1)}{\partial \xi^{2}}=0, \\
& \frac{\partial^{3} V(1)}{\partial \xi^{3}}+k^{2} \frac{\partial V(1)}{\partial \xi}=0
\end{aligned}
$$

The similar procedure to tension case is repeated here to find out eigenfrequencies and mode shapes for the compression case.

\section{References}

[1] E.S. Hung, S.D. Senturia, Extending the travel range of analog-tuned electrostatic actuators, J. Microelectromech. Syst. 8 (1999) 497-505.

[2] E.M. Abdel-Rahman, M.I. Younis, A.H. Nayfeh, Characterization of the mechanical behavior of an electrically actuated microbeam, J. Micromech. Microeng. 12 (2002) 759-766.

[3] H.A. Tilmans, R. Legtenberg, Electrostatically driven vacuumencapsulated polysilicon resonators: part II. Theory and performance, Sens. Actuators A 45 (1994) 67-84.

[4] Y. Ahn, H. Guckel, J.D. Zook, Capacitive microbeam resonator design, J. Micromech. Microeng. 11 (2001) 70-80.
[5] H. Toshiyoshi, W. Piyawattanametha, C.T. Chan, M.C. Wu, Linearization of electrostatically actuated surface micromachined 2D optical scanner, J. Microelectromech. Syst. 10 (2001) 205-214.

[6] B. Choi, E.G. Lovell, Improved analysis of microbeams under mechanical and electrostatic loads, J. Micromech. Microeng. 7 (1997) 2429.

[7] H.C. Nathanson, W.E. Newell, R.A. Wickstrom, J.R. Davis, The resonant gate transistor, IEEE Trans. Electron Dev. ED-14 (1967) 117133.

[8] J.I. Seeger, S.B. Crary, Stabilization of electrostatically actuated mechanical devices, TRANDUCERS'97, International Conference on SolidState Sensors and Actuators, Chicago, IL, 1997, pp. 1133-1136.

[9] P.M. Osterberg, S.D. Senturia, M-TEST: A test chip for MEMS material property measurement using electrostatically actuated test structures, J. Microelectromech. Syst. 6 (1997) 107-118.

[10] O. Bochobza-Degani, Y. Nemirovsky, Modeling the pull-in parameters of electrostatic actuators with a novel lumped two degrees of freedom pull-in model, Sens. Actuators A 97-98 (2002) 569-578.

[11] O. Bochobza-Degani, Y. Nemirovsky, Experimental verification of a design methodology for torsion actuators based on a rapid pull-in solver, J. Microelectromech. Syst. 13 (2004) 121-130.

[12] G.D. Gray, M.J. Morgan, P.A. Kohl, Electrostatic actuators with expanded tuning range due to biaxial intrinsic stress gradients, J. Microelectromech. Syst. 13 (2004) 51-62.

[13] Y. Nemirovsky, O. Bochobza-Degani, A methodology and model for the pull-in parameters of electrostatic actuators, J. Microelectromech. Syst. 10 (2001) 601-615.

[14] S.D. Senturia, Microsystem Design, Kluwer Academic, Boston, MA, 2001.

[15] L.D. Gabby, J.E. Mehner, S.D. Senturia, Computer-aided generation of nonlinear reduced-order dynamic macromodels-II: non-stress-stiffened case, J. Microelectromech. Syst. 9 (2000) 262-269.

[16] J.E. Mehner, L.D. Gabby, S.D. Senturia, Computer-aided generation of nonlinear reduced-order dynamic macromodels-I: stress-stiffened case, J. Microelectromech. Syst. 9 (2000) 270-277.

[17] L.X. Zhang, Y.P. Zhao, Electromechanical model of RF MEMS switches, Microsyst. Technol. 9 (2003) 420-426.

[18] Y.C. Hu, C.M. Chang, S.C. Huang, Some considerations on the electrostatically actuated microstructures, Sens. Actuators A 112 (2004) $155-161$.

[19] S. Pamidighantam, R. Puers, K. Baert, H. Tilman, Pull-in voltage analysis of electrostatically actuated beam structures with fixed-fixed and fixed-free end conditions, J. Micromech. Microeng. 12 (2002) 458-464.

[20] N. Lobontiu, E. Garcia, Two microcantilever designs: lumped-parameter model for static and modal analysis, J. Microelectromech. Syst. 13 (2004) 41-50.

[21] G.W. Wang, Y. Zhang, Y.P. Zhao, G.T. Yang, Pull-in instability study of carbon nanotube tweezers under the influence of van der Waals forces, J. Micromech. Microeng. 14 (2004) 1119-1125.

[22] O. Bochobza-Degani, D. Elata, Y. Nemirovsky, An efficient DIPIE algorithm for CAD of electrostatically actuated MEMS devices, J. Microelectromech. Syst. 11 (2002) 612-620.

[23] H. Rong, Q.A. Huang, M. Nie, W. Li, An analytical model for pull-in voltage of clamped-clamped multilayer beams, Sens. Actuators A 116 (2004) $15-21$.

[24] X.M. Zhang, F.S. Chau, C. Quan, Y.L. Lam, A.Q. Liu, A study of the static characteristics of a torsional micromirror, Sens. Actuators A 90 (2001) 73-81.

[25] G.A. Korn, T.M. Korn, Mathematical Handbook for Scientist and Engineers, second ed., McGraw-Hill Book Company, 1968.

[26] T. Chang, R.R. Craig Jr., Normal modes of uniform beam, J. Eng. Mech. Div., ASCE 95 (1969) 1027-1031.

[27] A. Chajes, Principles of Structural Stability Theory, Prentice-Hall Inc., 1974.

[28] S. Timoshenko, S. Woinosky-Krieger, Theory of Plates and Shells, second ed., McGraw-Hill Book Company Inc., 1959.

[29] D.J. Gorman, Free Vibration Analysis of Rectangular Plates, Elsevier North Holland Inc., 1982. 


\section{Biographies}

Yin Zhang, BS, Department of Mechanics, Peking University, Beijing, China, 1997 and PhD, Department of Mechanical Engineering, University of Connecticut, Storrs, Connecticut, USA, 2003. From 2003 to 2004, he worked as an assistant professor in the State Key Laboratory of Nonlinear Mechanics (LNM), Institute of Mechanics, Chinese Academy of Sciences (CAS) and as an associate professor since 2004. His current research focuses on the MEMS/NEMS structural mechanics, dynamics and interface properties.
Ya-pu Zhao received the PhD degree from the Department of Mechanics, Peking University, Beijing, China, in 1994. From 1994 to 1996, he was a postdoctoral associate with the Institute of Mechanics, Chinese Academy of Sciences (CAS). Since 1996, he has been working in the State Key Laboratory of Nonlinear Mechanics (LNM), Institute of Mechanics, CAS, and was promoted to full professor in 1998. He is currently the Director of LNM, CAS. His current technical interests include stiction, anti-stiction (application of SAMs), bio-adhesion, surface forces, materials and structural analysis, design, and failure analysis of MEMS. 